\title{
TOPOLOGICAL RADICALS, I. BASIC PROPERTIES, TENSOR PRODUCTS AND JOINT QUASINILPOTENCE
}

\author{
VICTOR S. SHULMAN \\ Vologda Polytechnical Institute \\ Lenin St. 15, Vologda 160000, Russia \\ E-mail: shulman_v@yahoo.com \\ YURI V. TUROVSKII \\ Institute of Mathematics and Mechanics, National Academy of Sciences of Azerbaijan \\ F. Agaev St. 9, Baku AZ1141, Azerbaijan \\ E-mail: yuri@sinam.net
}

Dedicated to Professor Wiestaw Żelazko on the occasion of his 70-th birthday

\begin{abstract}
The paper starts a series of publications devoted to the theory of topological radicals (TRs) of normed algebras. It contains results on general properties of TRs and their domains, considers TRs close to the Jacobson radical, presents a theory of tensor TRs (they are connected with the problem of calculating the radical of a projective tensor product), introduces and studies TRs related to the notion of joint qusinilpotence.
\end{abstract}

1. Introduction. Studying some property, say $\mathcal{P}$, of associative algebras it is sometimes possible to single out the smallest ideals that accumulate $\mathcal{P}$ (or the largest ideals of algebras that have $\mathcal{P}$ ). This means that the quotients by these ideals are free of $\mathcal{P}$ in the sense that they do not have nonzero ideals that possess $\mathcal{P}$. Thus one obtains two, with respect to $\mathcal{P}$, classes of algebras (usually called $\mathcal{P}$-radical and $\mathcal{P}$-semisimple) which can be investigated separately and then joined by means of the extension theory. The first important examples were related to the nilpotence and some close properties, namely lower and upper nil radicals, quasi-regular radical, etc. The intensive development of this approach brought to a rich and fruitful branch of the modern algebra, the general theory of radicals $[5,22]$, as maps associating to an algebra its ideal and satisfying some special axioms.

2000 Mathematics Subject Classification: Primary 46H20, 46H15; Secondary 46B25, 46B45, $46 \mathrm{H} 40$.

Key words and phrases: Banach algebra, normed algebra, $Q$-algebra, topological radical, tensor product, topologically nil ideal, joint spectral radius.

The paper is in final form and no version of it will be published elsewhere. 
A topological analog of this theory, namely the theory of topological radicals of normed algebras, was initiated by P. G. Dixon [7]. This work contained a well thought-out axiomatics, topological versions of some basic constructions and many interesting examples. What is especially important, [7] proposed a radical theory approach to one of difficult problems of the Banach algebra theory, the problem of the existence of topologically irreducible representations of Jacobson radical Banach algebras. The solution of this problem, obtained by C. Read [16], was stimulated by Dixon's approach.

Here we start a series of works on topological radicals of normed (in particular Banach) algebras. They are related to some known problems of Banach algebra theory and operator theory: the existence of non-trivial ideals, radicality of tensor products, joint quasinilpotence of topologically nil algebras, invariant subspaces, scarcity of spectra, spectral theory of multiplication operators and so on. Most of them will be completely settled only in the presence of the (weakest possible) compactness type conditions, but many partial results will be obtained without such assumptions. We are aimed also in the intrinsic development of the theory, not related directly to the outer problems.

The present paper consists of three sections. The first one, Section 2, considers the basic properties of topological radicals (TRs) and their domains, the ground and universal classes of normed algebras. We establish some useful general properties of TRs, classify TRs with respect to some more special conditions, present, compare and investigate examples of TRs related to the Jacobson radical (which itself is not a TR if considered on the class of all normed algebras). It is worth mentioning that the developed theory of TRs finds its first applications in the difficult problem of distinguishing some universal classes (see Subsection 2.9). A part of the section is devoted to the study of extensions. They are understood in two different senses: the stability of a ground class with respect to the forming of extensions (does an algebra belongs to the ground class if this class contains its ideal and the quotient?) and the possibility to extend a TR to a larger ground class. The first direction is of technical use (but some results seem to be valuable themself, for example the extension stability of the class of all $Q$-algebras). The second one is of central importance and is related to many further topics of our project (starting with tensor products). The situation can be described as follows: dealing with a radical on a ground class it is very useful to know if it can be extended (with preservation of some special properties) to wider (presumably universal) classes. This (apart from the intrinsic beauty of the subject) justifies our interest in non-complete algebras: the class of Banach algebras is not universal.

Section 3 considers the behavior of a TR with respect to the forming of projective tensor products of Banach algebras. It was stimulated by the unsolved problem of radicality of a tensor product of a (Jacobson) radical Banach algebra and an arbitrary one. We define the general class of tensor TRs, and construct the tensor radical $R^{t}$ related to a given TR $R$ (coinciding with $R$ iff $R$ is tensor). More precisely we find some conditions under which $R^{t}$ is a TR and satisfies some additional properties. Then we restrict our attention to the case that $R=\operatorname{Rad}$, the Jacobson radical on the class of all Banach algebras, describe the properties of $\operatorname{Rad}^{t}$ and relate it with a kind of joint quasinilpotence (with respect to an $l^{1}$-version of the joint spectral radius). 
Recall that a bounded subset $M$ of a normed algebra is called jointly quasinilpotent if $\left\|M^{k}\right\|^{1 / k} \rightarrow 0$ as $k \rightarrow \infty$, where $M^{k}$ is the set of all products of $k$ elements from $M$ and the norm of a set is defined as supremum of norms of its elements. It is an open problem if in a radical Banach algebra each finite (or each precompact) set is jointly quasinilpotent. In Section 4 we construct and study topological radicals related to the properties of joint quasinilpotence and investigate their connections to $\operatorname{Rad}^{t}$. In subsequent publications we will return to these notions and problems.

The authors would like to express their gratitude to Maria Fragoulopoulou for a consultation on $Q$-algebras.

1.1. Preliminaries. In what follows all linear spaces and algebras are complex. For a linear space $X, L(X)$ denotes the algebra of linear operators on $X$. If $X$ is normed, $B(X)$ denotes the subalgebra of $L(X)$ consisting of all bounded operators on $X$. The completion of a normed space $X$ is denoted by $\bar{X}$. If $X$ is a subspace of a normed space $Y$ then $\bar{X}$ is identified as a rule with the closure of $X$ in $\bar{Y}$. So the closure of $X$ in $Y$ can be written as $\bar{X} \cap Y$.

The 'unitization' $A^{1}$ of an algebra $A$ is defined as $A$ itself if $A$ is unital and as $A \oplus \mathbb{C}$, with standard operations, otherwise. The term 'ideal' means a two-sided ideal; note that all ideals of $A$ are simultaneously ideals of $A^{1}$. If $I$ is an ideal of $A$ then $q_{I}$ denotes the canonical epimorphism of $A$ onto the quotient $A / I$. Sometimes instead of $q_{I}(a)$ (resp. $\left.q_{I}(M)\right)$ we write $a / I(\operatorname{resp} . M / I)$ for every $a \in A(\operatorname{resp} . M \subset A)$.

Let irr $A$ denote the set of all strictly irreducible representations of $A$. If $A$ is normed, let $\operatorname{irr}_{b} A$ (resp. $\operatorname{irr}_{n} A$ ) denote the set of all continuous representations in $\operatorname{irr} A$ by bounded operators on Banach (resp. normed) spaces. Two representations $\pi$ and $\tau$ in $\operatorname{irr} A$ on $X_{\pi}$ and $X_{\tau}$, respectively, are called equivalent if there exists an isomorphism $T: X_{\pi} \rightarrow X_{\tau}$ such that $\tau(a) T=T \pi(a)$ for every $a \in A$. It is known that any strictly irreducible representation of an algebra $A$ is equivalent to the left regular representation $\pi^{M}$ on the quotient space $A / M$, where $M$ is a maximal modular left ideal. Let Prim $A$ denote the set of primitive ideals of $A$ (i.e. the kernels of representations in $\operatorname{irr} A$ ). By definition, the Jacobson radical $\operatorname{rad}(A)$ is the intersection of all ideals in Prim $A^{1}$. There are many equivalent algebraic characterizations of $\operatorname{rad}(A)$; in particular it is equal to the intersection of all modular maximal left ideals. Also, $\operatorname{rad}(A)$ is the largest quasi-regular ideal (i.e. all its elements are quasi-invertible; $a \in A$ is quasi-invertible if $1-a$ is invertible in $A^{1}$ ).

The spectrum $\sigma_{A}(a)$ of an element $a \in A$ is the set of all $\lambda \in \mathbb{C}$ for which $a-\lambda$ is not invertible in $A^{1}$ (this definition agrees really with one via quasi-inverses in [13, Section 2.1]). The algebraic spectral radius $\rho_{A}(a)$ is defined as $\sup \left\{|\lambda|: \lambda \in \sigma_{A}(a)\right\}$. If $A$ is a normed algebra then $\sigma_{A}(a)$ is always nonempty ([13, Theorem 2.2.2]). In this case one defines also the topological spectral radius $\rho(a)=\lim \left\|a^{n}\right\|^{1 / n}=\inf \left\|a^{n}\right\|^{1 / n}$. In general $\rho(a) \leqslant \rho_{A}(a)$ and $\rho(a)=\rho_{\bar{A}}(a)$ by Gelfand's spectral radius formula, where $\bar{A}$ is the completion of $A$. If $\rho(a)=0$, we say that $a$ is quasinilpotent. A (one-sided) ideal is topologically nil [13] if it consists of quasinilpotent elements.

\section{Topological radicals, classes of normed algebras, and the Jacobson radical.}

In this section we investigate the properties of TRs defined on various classes of normed algebras. First of all we consider normed $Q$-algebras. 
2.1. Q-algebras. A normed algebra $A$ is called a $Q$-algebra if the set of all invertible elements of $A^{1}$ is open. Several equivalent characterizations of this important property can be found in [13]. In particular, the following are equivalent [13, Proposition 2.2.7] for normed algebras.

$\left(\mathrm{Q}_{1}\right) \quad A$ is a $Q$-algebra.

$\left(\mathrm{Q}_{2}\right) \quad \rho_{A}(a)=\rho(a)$ for any $a \in A$.

(Q3) $\quad \sum_{n>0} a^{n}$ converges for any $a \in A$ with $\|a\|<1$.

It follows that in any $Q$-algebra the norm is spectral, i.e., $\rho_{A}(a) \leqslant\|a\|$; conversely, an algebra with spectral norm is a $Q$-algebra. If $A$ is a subalgebra of an algebra $B, A$ is called a spectral subalgebra of $B$ [13, Definition 2.5.1] if $\sigma_{A}(a) \backslash\{0\}=\sigma_{B}(a) \backslash\{0\}$ for every $a \in A$.

Our aim here is to prove that $Q$-algebras can be conveniently characterized in terms of their strictly irreducible representations.

TheOREM 2.1. For a normed algebra $A,\left(\mathrm{Q}_{1}\right)$ is equivalent to the following conditions.

$\left(\mathrm{Q}_{4}\right) \quad$ Each maximal modular left ideal of $A$ is closed.

$\left(\mathrm{Q}_{5}\right) \quad$ Each strictly irreducible representation of $A$ is equivalent to a continuous representation (by bounded operators on a normed space).

Proof. The implication $\left(\mathrm{Q}_{1}\right) \Rightarrow\left(\mathrm{Q}_{4}\right)$ is well known [4, Theorem 2.2.8]. $\left(\mathrm{Q}_{4}\right) \Rightarrow\left(\mathrm{Q}_{5}\right)$ follows from the fact that any strictly irreducible representation of $A$ is equivalent to some representation $\pi^{M}$, where $M$ is a maximal modular left ideal of $A$. If $M$ is closed then the space $A / M$ obtains the quotient norm with respect to which $\pi^{M}$ is clearly continuous.

Now let $\left(\mathrm{Q}_{5}\right)$ hold; we will show $\left(\mathrm{Q}_{5}\right) \Rightarrow\left(\mathrm{Q}_{2}\right)$. It suffices to prove that $A$ is a spectral subalgebra of $\bar{A}$. Let $a \in A$ and, for some nonzero $\lambda \in \mathbb{C}, a-\lambda$ be non-invertible in $A^{1}$. We should prove that it is not invertible in $B^{1}$, where $B=\bar{A}$.

If $a-\lambda$ is not left invertible in $A^{1}$ then there is a maximal left ideal $M$ of $A^{1}$ containing $a-\lambda$. Let $x=1+M \in A^{1} / M$. Then

$$
\pi^{M}(a) x=\lambda x .
$$

Since $\pi^{M}(A) \neq 0$, the restriction of $\pi^{M}$ to $A$ is strictly irreducible. We proved that there is a strictly irreducible representation $\pi$ of $A$ such that $\lambda$ is an eigenvalue of $\pi(a)$. By the assumption, we can suppose that $\pi$ is a continuous representation by bounded operators on a normed space $X$. Let $Y=\bar{X}$, the completion of $X$, and let $\bar{\pi}$ be the representation of $A$ on $Y$ such that $\bar{\pi}(a)$ is the extension of $\pi(a)$ by continuity, for any $a \in A$. Again $\bar{\pi}$ extends by continuity to a representation $\tau$ of $B$ which in its turn extends to a representation $\tau^{\prime}$ of $B^{1}$ on the same space. It is easy to see that $\lambda$ is an eigenvalue of $\tau^{\prime}(a)$. But this means that the element $a$ cannot be left invertible in $B^{1}$.

So we may suppose that $a-\lambda$ is left invertible in $A^{1}$ :

$$
c(a-\lambda)=1
$$

for some $c \in A^{1}$. If $a-\lambda$ is invertible in $B^{1}$ then

$$
(a-\lambda) b=1
$$


for some $b \in B^{1}$, whence

$$
c=c(a-\lambda) b=b
$$

and accordingly $b \in A^{1}$, a contradiction. This shows that $A$ is a spectral subalgebra of $\bar{A}$.

We continue the list of conditions equivalent to $\left(\mathrm{Q}_{1}\right)$.

(Q6) $A$ is a spectral subalgebra of $\bar{A}$.

$\left(\mathrm{Q}_{7}\right)$ Every strictly irreducible representation of $A$ extends to a representation of $\bar{A}$.

The equivalence of the conditions $\left(\mathrm{Q}_{6}\right)$ and $\left(\mathrm{Q}_{7}\right)$ is a special case of Schweitzer's Theorem [13, Theorem 4.2.10], the equivalence of $\left(\mathrm{Q}_{6}\right)$ and $\left(\mathrm{Q}_{1}\right)$ is well known (for instance see [12, Lemma 20.9]).

Looking at the condition $\left(\mathrm{Q}_{5}\right)$ of Theorem 2.1 as a possible definition of a $Q$-algebra it is natural to consider normed algebras whose strictly irreducible representations are equivalent to continuous representations (by bounded operators) on Banach spaces. We call them $Q_{b}$-algebras. Being related to a special radical introduced in [7], these algebras play an important role in what follows.

2.2. Classes of normed algebras. Let $(N A)$ denote the class of all normed algebras. The following definitions will be useful.

A class $\mathcal{A} \subset(N A)$ is called image closed (resp. preimage closed) if conditions $B=$ $f(A)$ for a continuous isomorphism $f$ and $A \in \mathcal{A}$ (resp. $B \in \mathcal{A}$ ) imply $B \in \mathcal{A}$ (resp. $A \in \mathcal{A}$ ). Recall that a continuous isomorphism is a topological isomorphism if its inverse is also continuous.

A class $\mathcal{A} \subset(N A)$ is called ideal stable (resp. closed ideal stable) if it contains all normed algebras topologically isomorphic to ideals (resp. closed ideals) of every $A \in \mathcal{A}$. A class $\mathcal{A} \subset(N A)$ is called quotient stable if it contains all normed algebras topologically isomorphic to quotients of every $A \in \mathcal{A}$ by closed ideals.

A class $\mathcal{A} \subset(N A)$ is called a ground class if it is closed ideal and quotient stable, and a universal class if it is ideal and quotient stable. Note that ground and universal classes of normed algebras are natural domains of topological radicals.

In many cases the properties of synthetic kind are important. For $\mathcal{A} \subset \mathcal{B} \subset(N A), \mathcal{A}$ is called extension stable in $\mathcal{B}$ if conditions $A \in \mathcal{B}, I$ is a closed ideal of $A$ and $A / I, I \in \mathcal{A}$ imply $A \in \mathcal{A}$. We say that $\mathcal{A}$ is linear in $\mathcal{B}$ if every $A \in \mathcal{B}$ with a dense sum of its ideals $I_{\alpha}$ that belong to $\mathcal{A}$ belongs to $\mathcal{A}$ itself. In both cases we do not mention $\mathcal{B}$ if $\mathcal{B}=(N A)$.

Let us now consider some examples of ground classes.

- The class $(B A)$ of all Banach algebras. It is an easy exercise that the class is extension stable, not linear and not ideal stable.

- The class $(Q A)$ (resp. $\left.\left(Q_{b} A\right)\right)$ of all normed $Q$-algebras (resp. $Q_{b}$-algebras). Classes $(Q A)$ and $\left(Q_{b} A\right)$ are universal (see Theorem 2.5).

- The smallest universal class $(B A)^{u}$ containing all Banach algebras.

- The class $(C N A)$ of all commutative normed algebras. The class is universal, not extension stable and not linear.

○ The class $\left(C^{*} E A\right)$ of all $C^{*}$-equivalent algebras. The class is not universal. We will investigate it elsewhere. 
Restricting "dimension" of algebras in a ground class we get another ground class. In this way one obtains the classes $(S B A)$ and $(S N A)$ of all separable Banach and, respectively, normed algebras and the class $(F N A)$ of all finite-dimensional normed algebras. There are many possibilities to form new ground classes starting from the given ones.

Proposition 2.2. Unions and intersections of arbitrary families of ground (resp. universal) classes are ground (resp. universal) classes.

Proof. Clear.

In particular the intersection of any ground class with $(C N A)$ is often important. It is easy to see that

$$
\left(C^{*} E A\right) \subset(B A) \subset(B A)^{u} \subset\left(Q A_{b}\right) \subset(Q A) \subset(N A) .
$$

2.3. Classes $(Q A)$ and $\left(Q_{b} A\right)$. The algebraic parts of the following two lemmas belong to the folklore and we are mainly interested in the topological aspect. Let $A$ be an algebra, and let $I$ be its ideal. Put $\operatorname{irr}_{I} A=\{\pi \in \operatorname{irr} A: \pi I=0\}$ and $\operatorname{irr}^{I} A=\{\pi \in \operatorname{irr} A: \pi I \neq 0\}$. Let $q_{I}$ be the standard epimorphism $A \rightarrow A / I$, and let $q_{I}^{-1}(a)$ be the preimage of $a \in A / I$.

Lemma 2.3. Let $A \in(N A)$, and let $I$ be its closed ideal. If $A$ belongs to $\left(Q_{b} A\right)$ (resp. $(Q A))$ then so does $A / I$. If $A / I \in\left(Q_{b} A\right)$ (resp. $(Q A)$ ) then every representation from $\operatorname{irr}_{I} A$ is equivalent to a continuous representation in $\operatorname{irr}_{I} A$ by bounded operators on a Banach (resp. normed) space.

Proof. Consider first $A$ and $I$ in the pure algebraic context (i.e. without any topology). For arbitrary $\pi \in \operatorname{irr} A / I$ and $\tau \in \operatorname{irr}_{I} A$, put $\widehat{\pi}=\pi q_{I}$ and $\widetilde{\tau}=\tau q_{I}^{-1}$. Clearly $\widehat{\pi} \in \operatorname{irr}_{I} A$ and $\widetilde{\tau} \in \operatorname{irr} A / I$. If $\tau^{\prime}$ is equivalent to $\tau$ and $\widetilde{\tau}$ is equivalent to $\pi$ then $\tau^{\prime}$ is equivalent to $\widehat{\pi}$. Indeed, identifying the representation spaces pairwise, one can identify the actions of $\tau^{\prime}$ and $\widehat{\pi}$. Similarly, if $\pi^{\prime}$ is equivalent to $\pi$ and $\widehat{\pi}$ is equivalent to $\tau$ then $\pi^{\prime}$ is equivalent to $\widetilde{\tau}$.

If $A$ is normed, $I$ is closed and $\pi$ (resp. $\tau$ ) is a bounded representation by bounded operators on a normed (or a Banach) space, we have that $\widehat{\pi}$ (resp. $\widetilde{\tau}$ ) is a representation by bounded operators and is bounded. Indeed, $\|\widehat{\pi}\| \leqslant\|\pi\|\left\|q_{I}\right\|$ and, for every $a \in A$,

$$
\|\widetilde{\tau}(a)\| \leqslant\|\tau\| \inf \left\{\|b\|: b \in q_{I}^{-1}(a)\right\} \leqslant\|\tau\|\|a\|,
$$

whence $\|\widetilde{\tau}\| \leqslant\|\tau\|$.

Lemma 2.4. Let $A \in(N A)$, and let I be its ideal. If $A$ belongs to $\left(Q_{b} A\right)$ (resp. $\left.(Q A)\right)$ then so does $I$. If $I \in\left(Q_{b} A\right)$ (resp. $(Q A)$ ) then every representation from $\operatorname{irr}^{I} A$ is equivalent to a continuous representation in $\operatorname{irr}^{I} A$ by bounded operators on a Banach (resp. normed) space.

Proof. Consider first $A$ and $I$ in the pure algebraic context. For arbitrary $\pi \in \operatorname{irr}^{I} A$ and $\tau \in \operatorname{irr} I$, let $\left.\pi\right|_{I}$ be the restriction of $\pi$ to $I$ and, for arbitrary nonzero $x \in X_{\tau}, y=\tau(b) x$ with $b \in I$, let $\tau_{x}$ be defined as $\tau_{x}(a) y=\tau(a b) x$ for every $a \in A$. The definition of $\tau_{x}$ is correct: if $y=0$ then $\tau(I) \tau(a b) x=\tau(I a) y=0$, whence $\tau(a b) x=0$. Clearly $\left.\pi\right|_{I} \in \operatorname{irr} I$ and $\tau_{x} \in \operatorname{irr}^{I} A$, and, for fixed $\tau$, all $\tau_{x}$ are pairwise equivalent. 
If $\pi^{\prime}$ is equivalent to $\pi$ and $\left.\pi\right|_{I}$ is equivalent to $\tau$ then $\pi^{\prime}$ is equivalent to $\tau_{x}$, for every $x$. Indeed, identifying $\pi^{\prime}$ with $\pi$ and $\tau$ with $\left.\pi\right|_{I}$, one can assume that the representations act on the same space, say $X$ and, for every $y=\tau(b) x=\left.\pi\right|_{I}(b) x=\pi(b) x \in X$ with $b \in I$ and for every $a \in A$, we have

$$
\tau_{x}(a) y=\tau(a b) x=\left.\pi\right|_{I}(a b) x=\pi(a b) x=\pi(a) y .
$$

Similarly, if $\tau^{\prime}$ is equivalent to $\tau$ and $\tau_{x}$ is equivalent to $\pi$ then $\tau^{\prime}$ is equivalent to $\left.\pi\right|_{I}$. Indeed, identifying $\tau^{\prime}$ with $\tau$ and $\tau_{x}$ with $\pi$, one can assume that the representations act on the same space, say $X$ and, for every $y=\tau(b) x \in X$ with $b \in I$ and for every $a \in I$, we have

$$
\left.\pi\right|_{I}(a) y=\pi(a) y=\tau_{x}(a) x=\tau(a b) x=\tau(a) y .
$$

If $A$ is normed and $\pi$ (resp. $\tau$ ) is a bounded representation by bounded operators on a normed (Banach) space $X$, we have that $\left.\pi\right|_{I}$ (resp. $\tau_{x}$ ) is a representation by bounded operators and is bounded. It suffices to check boundedness for $\tau_{x}$ if $\tau$ is bounded. For every $y \in X$, put

$$
\|y\|_{(I, x)}=\inf \{\|b\|: b \in I, \quad \tau(b) x=y\} .
$$

Then $y \mapsto\|y\|_{(I, x)}$ determines a new norm on $X$. Indeed, if $\|y\|_{(I, x)}=0$ then $\|y\|=$ $\|\tau(b) x\| \leqslant\|\tau\|\|b\|\|x\|$ implies $\|y\|=0$, i.e. $y=0$; the other properties of norm are obvious for $y \mapsto\|y\|_{(I, x)}$. We have that, for every $a \in A$,

$$
\left\|\tau_{x}(a) y\right\|_{(I, x)} \leqslant\|\tau(a b) x\|_{(I, x)} \leqslant\|a b\| \leqslant\|a\|\|b\|,
$$

whence

$$
\left\|\tau_{x}(a) y\right\|_{(I, x)} \leqslant\|a\|\|y\|_{(I, x)}, \quad\left\|\tau_{x}(a)\right\|_{(I, x)} \leqslant\|a\|, \quad\left\|\tau_{x}\right\|_{(I, x)} \leqslant 1 .
$$

If $X$ is a Banach space, take $\bar{I}$ instead of $I$ and $\bar{\tau}$, the continuous extension of $\tau$ to $\bar{I}$ by bounded operators on $X$, instead of $\tau$. Then the above estimates hold, and it remains to show that $\left(X,\|\cdot\|_{(\bar{I}, x)}\right)$ is complete. Indeed, $\left(X,\|\cdot\|_{(\bar{I}, x)}\right)$ is isometrically isomorphic to the quotient $\bar{I} / M$, where $M=\{b \in \bar{I}: \bar{\tau}(b) x=0\}$, and hence is complete.

Now we are in a position to describe the basic properties of $\left(Q_{b} A\right)$ and $(Q A)$.

TheOREm 2.5. $\left(Q_{b} A\right)$ and $(Q A)$ are extension stable, preimage closed, linear universal classes.

Proof. It follows from Lemmas 2.3 and 2.4 that $\left(Q_{b} A\right)$ and $(Q A)$ are extension stable and universal.

Let $A \in(N A), B \in(Q A)$ and $f(A)=B$ for some continuous isomorphism $f$. Then

$$
\rho(a) \geqslant \rho(f(a))=\rho_{B}(f(a))=\rho_{A}(a)
$$

and therefore $\rho_{A}(a)=\rho(a)$ for every $a \in A$. Thus $A \in(Q A)$.

Suppose now that $B \in\left(Q_{b} A\right)$. Take an arbitrary $\pi \in \operatorname{irr} A$. Then the representation $\pi f^{-1} \in \operatorname{irr} B$ is equivalent to some $\tau \in \operatorname{irr}_{b} B$. Hence $\tau f \in \operatorname{irr}_{b} A$. Indeed, $\tau f(a) x=$ $\tau(f(a)) x$ for every $a \in A$ and $x \in X_{\tau}$, whence $\tau f(a) \in B\left(X_{\tau}\right)$ and also $\|\tau f\| \leqslant\|\tau\|\|f\|$. It is easy to see that $\pi$ is equivalent to $\tau f$. So $A \in\left(Q_{b} A\right)$. This shows that $\left(Q_{b} A\right)$ and $(Q A)$ are preimage closed. 
Let $A \in(N A)$ be the closure of sum of its ideals $I_{\alpha} \in\left(Q_{b} A\right)$ (resp. $(Q A)$ ), and let $\pi \in \operatorname{irr} A$ be arbitrary. Then there exists an index $\beta$ such that $\pi I_{\beta} \neq 0$. By Lemma $2.4, \pi$ is equivalent to a continuous representation $\operatorname{in}^{\operatorname{irr}^{I_{\beta}}} A$ by bounded operators on a Banach (resp. normed) space. Therefore $A \in\left(Q_{b} A\right)$ (resp. $\left.(Q A)\right)$. We proved that $\left(Q_{b} A\right)$ and $(Q A)$ are linear.

The facts that $(Q A)$ is universal and preimage closed are known [13]. Also, I. Kaplansky [11, Lemma 3] proved that $(Q A)$ is extension stable. An ideal of a normed algebra is called a $Q$-ideal (resp. $Q_{b}$-ideal) if it is a $Q$-algebra (resp. $Q_{b}$-algebra).

COROLlARY 2.6. Every normed algebra has the largest $Q$-ideal (resp. $Q_{b}$-ideal); the latter is closed.

Proof. Let $A \in(N A)$ and let $J$ be the closure of sum of all $Q$-ideals (resp. $Q_{b}$-ideals) $I_{\alpha}$ of $A$. Then every $I_{\alpha}$ is also an ideal of $J$. It follows from Theorem 2.5 that $J \in(Q A)$ (resp. $\left.\left(Q_{b} A\right)\right)$.

Extension stability and linearity of $(Q A)$ solve in the context of normed algebras some Palmer's questions on spectral algebras [13, Page 234].

2.4. Topological radicals and their elementary properties. Recall that an epimorphism $f: A \rightarrow B$ of normed algebras is open (i.e., the images of open sets are open) iff there is a constant $C>0$ such that for any $b \in B$ there is $a \in A$ with $f(a)=b$ and $\|a\| \leqslant C\|b\|$. An example of an open continuous epimorphism is a quotient map $q_{I}: A \rightarrow A / I$, where $I$ is a closed ideal of $A$. It is not difficult to check that any open continuous epimorphism $f$ is the composition of a topological isomorphism and the quotient map $q_{\text {ker } f}$.

Let $R$ be a map associating with any algebra $A$ in a ground class $\mathcal{A}$ its closed ideal $R(A)$. It is called a topological radical on $\mathcal{A}(\mathcal{A}$-radical, for short) if the following conditions are satisfied.

$\left(1^{\circ}\right) \quad R(R(A))=R(A)$, for any $A \in \mathcal{A}$.

$\left(2^{\circ}\right) \quad R(A / R(A))=0$, for any $A \in \mathcal{A}$.

$\left(3^{\circ}\right) \quad f(R(A))=R(B)$, for any topological isomorphism $f: A \rightarrow B$ with $A, B \in \mathcal{A}$.

$\left(4^{\circ}\right) \quad q_{I}(R(A)) \subset R(A / I)$, for any closed ideal $I$ of $A \in \mathcal{A}$.

$\left(5^{\circ}\right)$ If an ideal $I$ of $A \in \mathcal{A}$ belongs to $\mathcal{A}$ then

$\left(5_{1}^{\circ}\right) \quad R(I)$ is an ideal of $A$.

$\left(5_{2}^{\circ}\right) \quad R(I) \subset I \cap R(A)$.

If the class $\mathcal{A}$ is obvious we simply say that $R$ is a topological radical (TR). A TR $R$ is called a hereditary topological radical (HTR) if it satisfies the following condition stronger than $\left(5^{\circ}\right)$ :

$\left(6^{\circ}\right) \quad R(I)=I \cap R(A)$, for any ideal $I \in \mathcal{A}$ of $A \in \mathcal{A}$.

If $R$ satisfies the conditions $\left(1^{\circ}\right),\left(3^{\circ}\right),\left(4^{\circ}\right)$ and $\left(5^{\circ}\right)$ (respectively $\left.\left(2^{\circ}\right),\left(3^{\circ}\right),\left(4^{\circ}\right),\left(5^{\circ}\right)\right)$ then it is called an under topological radical (UTR) (respectively over topological radical (OTR)). If $\left(6^{\circ}\right)$ holds then a UTR is also called hereditary (note that a hereditary OTR is really an HTR).

The terms UTR and OTR were suggested by P. G. Dixon in virtue of [7, Theorem 6.11]; [7, Theorems 6.6 and 6.10] also clarified the reason for this terminology (note that 
the prefixes 'lower' and 'upper' are overloaded, while the prefixes 'sub' and 'super' usually mean something else).

Let $R$ be an $\mathcal{A}$-radical. An algebra $A \in \mathcal{A}$ is called $R$-semisimple if $R(A)=0 ; A$ is $R$-radical if $R(A)=A$. Clearly the ideals of $R$-semisimple algebras are $R$-semisimple and quotients of $R$-radical algebras by closed ideals are $R$-radical. If $R$ is an HTR then the ideals of an $R$-radical algebra are $R$-radical. The converse is always true: any $R$-radical ideal of $A$ is contained in $R(A)$. This can be formulated in the following way.

Lemma 2.7. Let $R$ be a TR on a ground class $\mathcal{A}$. Then, for each $A \in \mathcal{A}$,

(i) $R(A)$ is the largest $R$-radical ideal of $A$.

(ii) $\quad R(A)$ is the smallest closed ideal of $A$ with $R$-semisimple quotient.

Proof. (i) If $R(I)=I$ then the inclusion $I \subset R(A)$ follows from $\left(5^{\circ}\right)$.

(ii) If $R(A / I)=0$ then, by $\left(4^{\circ}\right), q_{I}(R(A))=0$ and $R(A) \subset I$.

Corollary 2.8. Let $R$ be a TR on a ground class $\mathcal{A}$.

(i) The closure of an $R$-radical ideal is $R$-radical.

(ii) If $I$ is a closed $R$-radical ideal of $A$ then $q_{I}(R(A))=R(A / I)$.

Proof. Let $I$ be an $R$-radical ideal of an algebra $A \in \mathcal{A}$, and let $J$ be the closure of $I$ in $A$. Then $J$ is a closed ideal of $A$, in particular $J \in \mathcal{A}$, and $I$ is an $R$-radical ideal of $J$. Since $R(J)$ is closed in $J$ and contains an ideal dense in $J, R(J)=J$. This proves (i).

For (ii), suppose that $I$ is a closed $R$-radical ideal of $A$. Then $I \subset R(A)$ and there is an open continuous epimorphism $p: A / I \rightarrow A / R(A)$ such that $q_{R(A)}=p q_{I}$. Hence $p(R(A / I)) \subset R(A / R(A))=0$, whence $R(A / I) \subset \operatorname{ker} p=q_{I}(R(A))$. The converse inclusion follows from $\left(4^{\circ}\right)$.

The following results on radicals will be useful.

TheOREM 2.9. Let $R$ be a TR on a ground class $\mathcal{A}$.

(i) The class of all $R$-semisimple (resp. R-radical) algebras is extension stable in $\mathcal{A}$.

(ii) If $A \in \mathcal{A}$ and every nonzero quotient of $A$ by a closed ideal contains a nonzero $R$-radical ideal then $A$ is $R$-radical.

Proof. (i) Let $I$ be a closed ideal of a normed algebra $A$.

Suppose that $A / I$ and $I$ are $R$-semisimple. Since $A / I$ is $R$-semisimple, $I$ contains $R(A)$ by Lemma 2.7. Hence $R(A)$ is an ideal of an $R$-semisimple algebra $I$. So $R(A)$ is $R$-semisimple, $R(A)=R(R(A))=0$.

Suppose that $A / I$ and $I$ are $R$-radical. Since $I$ is $R$-radical, it is contained in $R(A)$ by Lemma 2.7. Let $p: A / I \rightarrow A / R(A)$ be the open continuous epimorphism such that $q_{R(A)}=p q_{I}$. Then

$$
A / R(A)=p(A / I)=p(R(A / I)) \subset R(A / R(A))=0,
$$

whence $R(A)=A$.

(ii) If $A$ is not $R$-radical then $A / R(A)$ is $R$-semisimple and contains a nonzero $R$ radical, a contradiction. 
TheOREM 2.10. Let $R$ be a TR on a ground class $\mathcal{A}$.

(i) The class of all $R$-radical algebras is linear in $\mathcal{A}$.

(ii) If $\mathcal{A}$ is universal and $I$ is a sum of $R$-radical ideals $I_{\alpha}$ of $A \in \mathcal{A}$ then $I$ is $R$-radical. Proof. Let $I \in \mathcal{A}$ be the closure of a sum of $R$-radical ideals $I_{\alpha}$ in the case (i), or simply a sum of ones in the case (ii). In both cases $I \in \mathcal{A}$. Since $J_{\alpha}$ is an ideal of $I$, then $I_{\alpha}=R\left(I_{\alpha}\right) \subset R(I)$ for every $\alpha$. Therefore $R(I)$ is dense in $I$ (and also closed in $I$ ), whence $I=R(I)$.

TheOREM 2.11. Let $R$ be a TR on a ground class $\mathcal{A}$. Let $A \in \mathcal{A}$ be arbitrary, $I_{\alpha}$ closed ideals of $A$ and $I=\cap I_{\alpha}$. If $A / I_{\alpha}$ is $R$-semisimple for every $\alpha$ then $A / I$ is $R$-semisimple. Proof. Since $I \subset I_{\alpha}$, there exists an open continuous epimorphism $p_{\alpha}: A / I \rightarrow A / I_{\alpha}$ such that $q_{I_{\alpha}}=p_{\alpha} q_{I}$. By $\left(3^{\circ}\right)$ and $\left(4^{\circ}\right)$,

$$
p_{\alpha}(R(A / I)) \subset R\left(A / I_{\alpha}\right)=0,
$$

whence $R(A / I) \subset \operatorname{ker} p_{\alpha}=I_{\alpha} / I$ and $q_{I}^{-1}(R(A / I)) \subset q_{I}^{-1}\left(I_{\alpha} / I\right)=I_{\alpha}$ for every $\alpha$. So

$$
q_{I}^{-1}(R(A / I)) \subset \cap I_{\alpha}=I
$$

and accordingly $R(A / I)=0$.

We remark that the quotient of an $R$-semisimple algebra by a closed ideal need not be $R$-semisimple [2, Page 135], for a TR $R$.

2.5. Strong, strict and strictly hereditary radicals. The properties $\left(3^{\circ}\right)$ and $\left(4^{\circ}\right)$ describe the behavior of a TR under 'morphisms': they both can be written as

$\left(4_{f}^{\circ}\right) \quad f(R(A)) \subset R(B)$

whenever $B=f(A)$. In our setting morphisms are the compositions of topological isomorphisms and quotient maps, i.e. open continuous epimorphisms. It is sometimes reasonable to choose a wider class of morphisms, P. G. Dixon [7] considered all continuous epimorphisms as morphisms in $(N A)$. This increases the strength of a radical, but considerably reduces the list of radicals. On the other hand in some cases it is natural to consider more special morphisms, for example *-epimorphisms on the class $\left(C^{*} A\right)$ of all $C^{*}$-algebras. This class will be investigated later on.

Now we give some related definitions. Let us say that a TR $R$ is a strong radical on a ground class $\mathcal{A}$ if

$\left(7^{\circ}\right) \quad f(R(A)) \subset R(B)$ for each continuous epimorphism $f$ of algebras in $\mathcal{A}$.

We say that a TR $R$ is a strict radical on a ground class $\mathcal{A}$ if

$\left(8^{\circ}\right) \quad f(R(A))=R(B)$ for every continuous isomorphism $f: A \rightarrow B$ of algebras in $\mathcal{A}$.

It is not difficult to see that every strict radical is strong. It follows from the Open Mapping Theorem that all topological radicals on $(B A)$ are strict. The following result shows that for radicals on $(N A)$ this condition means actually the algebraic nature of a radical.

TheOREM 2.12. Let $R$ be a TR on the class $(N A)$. Then $R$ is strict iff $R$ does not depend on the choice of a norm, and iff $\left(8^{\circ}\right)$ holds for any algebraic isomorphism of normed algebras. 
Proof. Suppose that $R$ is strict. Let $\|\cdot\|_{1}$ and $\|\cdot\|_{2}$ be norms on an algebra $A$; we define a norm $\|\cdot\|$ on $A$ setting $\|a\|=\max \left(\|a\|_{1},\|a\|_{2}\right)$. Let $A, A_{1}$ and $A_{2}$ be the corresponding normed algebras. Taking the identity map as a continuous isomorphism from $A$ to $A_{m}$, $m=1,2$, we get

$$
R\left(A_{1}\right)=R(A)=R\left(A_{2}\right) .
$$

Therefore $R$ does not depend on a norm.

Now if $f: A \rightarrow B$ is an algebraic isomorphism of normed algebras then, denoting by $A_{f}$ the algebra $A$ with norm $\|a\|_{f}=\|f(a)\|$, we have that $f$ is a topological isomorphism from $A_{f}$ onto $B$ and

$$
R(B)=f\left(R\left(A_{f}\right)\right)=f(R(A)) .
$$

So $\left(8^{\circ}\right)$ holds for algebraic isomorphisms.

The converse is evident.

In some applications (for instance for extensions of radicals, tensor products etc.) it is useful to consider as a 'morphism' $A \rightarrow B$ a continuous homomorphism of $A$ onto an ideal of $B$. We need to introduce the corresponding definitions. A TR $R$ on a ground class $\mathcal{A}$ is called ideally strong if

$\left(7_{i}^{\circ}\right) \quad f(R(A)) \subset R(B)$ for each continuous epimorphism $f$ of an algebra $A \in \mathcal{A}$ onto an ideal of an algebra $B \in \mathcal{A}$.

Also, a TR $R$ on a ground class $\mathcal{A}$ is called strictly hereditary if

$\left(8_{i}^{\circ}\right) \quad f(R(A))=I \cap R(B)$ for each continuous isomorphism $f$ of an algebra $A \in \mathcal{A}$ onto an ideal $I$ of an algebra $B \in \mathcal{A}$.

Clearly every strictly hereditary TR is ideally strong. Also, every strictly hereditary TR is strict and hereditary (to see it, take $A=I$ and the identity map $f$ from $A$ onto $I$ in $\left(8_{i}^{\circ}\right)$, for an ideal $I$ of $B$ ), and every ideally strong TR is strong. The converse also holds if $\mathcal{A}$ is universal.

TheOREM 2.13. Let $R$ be a TR on a universal class $\mathcal{A}$. If $R$ is strong (resp. strict and hereditary) on $\mathcal{A}$ then $R$ is ideally strong (resp. strictly hereditary) on $\mathcal{A}$.

Proof. It is an easy checkup.

Recall that, by definition, a radical on the class of rings satisfies the axioms above in which topological restrictions are omitted (see [7, Section 6] and [5]; see also axioms of radicals in the sense of Amitsur and Kurosh in [22]).

THEOREM 2.14. Let $R$ be a radical (resp. hereditary radical) on the class all algebras. If $R(A)$ is closed for every algebra $A$ in a ground class $\mathcal{A} \subset(N A)$ then $R$ is an ideally strong $T R$ (resp. strictly hereditary $T R$ ) on $\mathcal{A}$.

Proof. Clear because $R$ satisfies axioms with algebraic 'morphisms' applied to the algebras from $\mathcal{A}$.

The most popular and important example of a hereditary radical on the class of all algebras (even rings) is the Jacobson radical rad. It should be stressed that rad is not a topological radical on $(N A)$, because there are normed algebras $A$ with non-closed $\operatorname{rad}(A)$ 
[7, Example 10.1]. For $Q$-algebras this obstacle disappears: since every maximal modular left ideal of a $Q$-algebra $A$ is closed, $\operatorname{rad}(A)$ is also closed. As a consequence, we obtain the following statement.

Corollary 2.15. rad is a strictly hereditary $T R$ on $(Q A)$.

Since a union of ground (resp. universal) classes is again ground (resp. universal), there exists a maximal ground (resp. universal) class on which rad is a TR. Let us denote it by $(\mathrm{rad})^{g}$ (resp. $\left.(\mathrm{rad})^{u}\right)$. It is interesting to explore the properties of these classes.

2.6. TRs connected with the Jacobson radical. Following the standard notation, we denote the restriction of $\mathrm{rad}$ to $(B A)$ by Rad. In the Banach algebra theory, Rad-semisimple (resp. Rad-radical) algebras are traditionally called semisimple (resp. radical). Now we consider two HTRs on $(N A)$ that coincide with rad on wider varieties of normed algebras.

Given an algebra $A \in(N A)$, let $\operatorname{rad}_{b}(A)\left(\operatorname{resp} . \operatorname{rad}_{n}(A)\right)$ be the intersection of the kernels of all representations in $\operatorname{irr}_{b}\left(A^{1}\right)\left(\operatorname{resp} . \operatorname{irr}_{n}\left(A^{1}\right)\right)$. It was shown in [7, Theorem 10.5] that $\operatorname{rad}_{b}$ is a strong HTR on $(N A)$ and all representations $\operatorname{in}_{\operatorname{irr}_{b}}(A)$ are strictly dense. We show the same for $\operatorname{rad}_{n}$. The following lemma generalizes [7, Theorem 10.2].

Lemma 2.16. For a normed space $X$, every strictly irreducible algebra $A \subset B(X)$ is strictly dense.

Proof. The proof of [3, Corollary 1.2.5.4] given for a Banach space is valid for a normed space.

As a consequence, any strictly irreducible representation of a (not necessarily topological) algebra by bounded operators on a normed space is strictly dense.

TheOREM 2.17. (i) $\operatorname{rad}_{n}$ is a strong HTR on $(N A)$.

(ii) For every $A \in(N A), \operatorname{rad}_{n}(A)$ contains every (one-sided, not necessarily closed) topologically nil ideal of $A$.

Proof. (i) Let $I$ be an ideal of a normed algebra $A$. The equality $\operatorname{rad}_{n}(I)=I \cap \operatorname{rad}_{n}(A)$ follows immediately from Lemma 2.4. Setting $I=\operatorname{rad}_{n}(A)$, we deduce $\operatorname{rad}_{n}\left(\operatorname{rad}_{n}(A)\right)=$ $\operatorname{rad}_{n}(A)$. Let again $I=\operatorname{rad}_{n}(A)$, and set $B=A / I$. It follows from Lemma 2.3 that if $b \in \operatorname{rad}(B)$ and $a \in b$ then $a \in I$, whence $b=0$. We proved that $B$ is $\operatorname{rad}_{n}$-semisimple.

It remains now to prove the equality $f\left(\operatorname{rad}_{n}(A)\right) \subset \operatorname{rad}_{n}(B)$, for any continuous epimorphism $f: A \rightarrow B$. But this immediately follows from the fact that $\pi f \in \operatorname{irr}_{n} A$ for every $\pi \in \operatorname{irr}_{n} B$.

(ii) Let $I$ be a topologically nil right ideal. Suppose that, for $\pi \in \operatorname{irr}_{n} A, b \in I$ and $x \in X_{\pi}, y=\pi(b) x \neq 0$. There exists $a \in A$ such that $\pi(a) y=x$, whence $\pi(b a) y=y$ and

$$
\|y\|^{1 / n}=\left\|\pi\left((b a)^{n}\right) y\right\|^{1 / n} \leqslant(\|\pi\|\|y\|)^{1 / n}\left\|(b a)^{n}\right\|^{1 / n} \rightarrow 0
$$

as $n \rightarrow \infty$, since $b a \in I$. So $y=0$, a contradiction. We obtain that $\pi I=0$ for every $\pi \in \operatorname{irr}_{n} A$.

If $I$ is a topologically nil left ideal, we use the equality $\pi(a b) x=x$ and the same argument as above.

TheOREM 2.18. $\operatorname{rad}_{b}=\operatorname{rad}$ on $\left(Q_{b} A\right)$ and $\operatorname{rad}_{n}=\operatorname{rad}$ on $(Q A)$. 
Proof. It is immediate (since equivalent representations have the same kernels).

Since $\operatorname{rad}(A)$ is a quasi-regular ideal, then, for a normed algebra $A, \operatorname{rad}(A)$ is a topologically nil ideal of $A$. As a consequence, we obtain the following well-known assertion.

Corollary 2.19. For an algebra $A \in(Q A), \operatorname{rad} A$ is the largest (one-sided; two-sided) topologically nil ideal of $A$. Moreover, the closure of a sum of topologically nil ideals of $A$ is a topologically nil ideal of $A$.

Proof. The statement follows from Theorems 2.17 and 2.18 (see also [13]).

2.7. Uniform TRs. An $\mathcal{A}$-radical $R$ is called uniform if all subalgebras of an $R$-radical algebra that belong to $\mathcal{A}$ are $R$-radical.

It follows that rad is a uniform HTR on $(Q A)$. It is also 'non-unital' in the sense that no unital algebra can be radical. We show that $\operatorname{rad}$ is the largest $\mathrm{TR}$ of $(Q A)$ radicals that share these properties. Note that, for a commutative normed algebra $A$ and a representation $\pi \in \operatorname{irr}_{n} A, \pi A$ is a normed division algebra and, by the Gelfand-Mazur Theorem, is one-dimensional.

Proposition 2.20. If $R$ is a uniform non-unital $(Q A)$-radical then $R(A) \subset \operatorname{rad}(A)$, for every $A \in(Q A)$.

Proof. Let us prove first that if a $Q$-algebra $A$ is $R$-radical then it is radical (i.e. $A=$ $\operatorname{rad}(A))$. If not then $A$ contains a non-quasinilpotent element and therefore there is a maximal non-radical commutative subalgebra $B$ of $A$. Since clearly $B$ is a spectral subalgebra of $A, B$ is a $Q$-algebra. There is a maximal ideal $I$ of $B$ with one-dimensional $B / I\left(I=\operatorname{ker} \pi\right.$ for some $\left.\pi \in \operatorname{irr}_{n} B\right)$. It follows that $B / I$ is unital and is not $R$-radical. Then $B$ is not $R$-radical that contradicts the assumption of uniformity of $R$.

Now for arbitrary $A \in(Q A)$ one has that $R(A)$ is radical and

$$
R(A)=\operatorname{rad}(R(A)) \subset \operatorname{rad}(A) .
$$

It should be noted that the same statement with actually the same proof holds for $(B A)$-radicals. In Section 4 we consider examples of topological radicals that are uniform on $(N A)$.

2.8. Regular TRs. We touch the important problem of extending of a TR to a wider ground class, in particular from $(B A)$ to $(N A)$. The following simple construction gives one of possible solutions for hereditary radicals.

Let $R$ be a map on a ground class $\mathcal{A} \supset(B A)$. For $A \in(N A)$ set

$$
R^{\prime}(A)=A \cap R(\bar{A}) \text {. }
$$

We call $R^{\prime}$ the regular extension of $R$ to $(N A) ; R$ is called regular on $\mathcal{A}$ if $R=R^{\prime}$ on $\mathcal{A}$. TheOREM 2.21. Let $\mathcal{A}$ be a ground class and $(B A) \subset \mathcal{A}$. If $R$ is a TR on $\mathcal{A}$ then $R^{\prime}$ is an $O T R$ on $(N A)$; if $R$ is an HTR on $\mathcal{A}$ then so is $R^{\prime}$ on $(N A)$.

Proof. Clearly $R^{\prime}(A)$ is a closed ideal in $A$, for every normed algebra $A$.

Let us first prove that, for an ideal $I$ of $A, R^{\prime}(I)$ is an ideal of $A$. Indeed, if $a \in R^{\prime}(I)$ and $b \in A$ then $a b \in I$ and $a b \in R(\bar{I})$, since $R(\bar{I})$ is an ideal of $\bar{A}$. So $a b \in R^{\prime}(I)$ and, 
similarly, $b a \in R^{\prime}(I)$. We have

$$
R^{\prime}(I)=I \cap R(\bar{I}) \subset I \cap R(\bar{A})=I \cap A \cap R(\bar{A})=I \cap R^{\prime}(A),
$$

so $\left(5^{\circ}\right)$ is proved.

For $\left(2^{\circ}\right)$, set $I=R^{\prime}(A)$. Identifying $A / I$ with $q_{\bar{I}}(A)$ (for $\left.q_{\bar{I}}: \bar{A} \rightarrow \bar{A} / \bar{I}\right)$, we identify $\overline{A / I}$ with $\bar{A} / \bar{I}$. Hence

$$
R^{\prime}(A / I)=q_{\bar{I}}(A) \cap R(\bar{A} / \bar{I})
$$

Since $\bar{I}$ is $R$-radical by Corollary 2.8(i), we obtain, by Corollary 2.8(ii), that $R(\bar{A} / \bar{I})=$ $q_{\bar{I}}(R(\bar{A}))$. Thus

$$
R^{\prime}(A / I)=q_{\bar{I}}(A) \cap q_{\bar{I}}(R(\bar{A}))=q_{\bar{I}}(A \cap R(\bar{A}))=q_{\bar{I}}(I)=0 .
$$

The second equality in the above chain follows from the inclusion $\bar{I} \subset R(\bar{A})$. Indeed if $q_{\bar{I}}(x) \in q_{\bar{I}}(A) \cap q_{\bar{I}}(R(\bar{A}))$, then there are elements $a, b \in \bar{I}$ such that $x+a \in A$, $x+b \in R(\bar{A})$. Hence $x+a \in R(\bar{A})+\bar{I}=R(\bar{A})$ and $q_{\bar{I}}(x)=q_{\bar{I}}(x+a) \in q_{\bar{I}}(A \cap R(\bar{A}))$.

The property $\left(3^{\circ}\right)$ is evident, and it remains only to prove $\left(4^{\circ}\right)$ : we have to show that

$$
q_{I}\left(R^{\prime}(A)\right) \subset R^{\prime}(A / I)
$$

for a closed ideal $I$ of $A$. Identifying $q_{I}(A)$ and $q_{I}\left(R^{\prime}(A)\right)$ with $q_{\bar{I}}(A) \subset \bar{A} / \bar{I}$ and $q_{\bar{I}}\left(R^{\prime}(A)\right)$ respectively, we obtain that

$$
\begin{aligned}
q_{I}\left(R^{\prime}(A)\right) & =q_{\bar{I}}\left(R^{\prime}(A)\right)=q_{\bar{I}}(R(\bar{A}) \cap A) \subset q_{\bar{I}}(R(\bar{A})) \cap q_{\bar{I}}(A) \\
& \subset R(\bar{A} / \bar{I}) \cap q_{\bar{I}}(A)=R^{\prime}(A / I) .
\end{aligned}
$$

We proved that $R^{\prime}$ is an OTR on $(N A)$.

Now let $R$ be an HTR. Then $R^{\prime}(I)=I \cap R^{\prime}(A)$ for every ideal $I$ of an algebra $A$. Indeed, since $R(\bar{I})=R(\bar{A}) \cap \bar{I}$, we have

$$
R^{\prime}(I)=R(\bar{I}) \cap I=R(\bar{A}) \cap \bar{I} \cap I=R(\bar{A}) \cap A \cap I=R^{\prime}(A) \cap I .
$$

Applying the proved equality to $I=R^{\prime}(A)$ we get $\left(1^{\circ}\right)$ :

$$
R^{\prime}\left(R^{\prime}(A)\right)=R^{\prime}(A) .
$$

Therefore $R^{\prime}$ is an HTR on $(N A)$.

Note that in general $R^{\prime}$ is not a strong TR on $(N A)$ even if $R$ is a strong HTR on $(B A)$ [7, Remark 10.8]. Let us say that an (NA)-radical $R$ is semi-regular if $R(A) \subset R(\bar{A})$. Then clearly the regular extension is the largest semi-regular extension for a given TR.

Following [7], let $T_{\infty}(A)$ (resp. $T_{m}(A)$, for $m \in \mathbb{N}$ ) be the intersection of the kernels of all continuous strongly dense (resp. topologically $m$-transitive) representations (of $A^{1}$ ) on Banach spaces. Recall that a continuous representation $\tau$ of $A$ on a Banach space $X$ is called topologically $m$-transitive if the map $a \mapsto\left(\tau(a) x_{1}, \ldots, \tau(a) x_{m}\right), a \in A$, has a dense image in the direct sum $X^{m}$ for every linearly independent system $x_{1}, \ldots, x_{m} \in X$, and strongly dense if this property holds for every $m \in \mathbb{N}$. Note that $T_{\infty}$ and $T_{m}$ are strong HTRs on $(N A)$ [7, Theorem 8.1], for every $m$.

Proposition 2.22. $T_{\infty}$ and $T_{m}$ are regular $H T R$ on $(N A)$, for every $m$.

Proof. For a normed algebra $A$, the maps $\pi \mapsto \bar{\pi}$, the continuous extension of $\pi$ of the algebra $A$ to $\bar{A}$, and $\left.\tau \mapsto \tau\right|_{A}$, the restriction of $\tau$ of the algebra $\bar{A}$ to $A$, determine 
bijections between the sets of continuous strongly dense (resp. topologically $m$-transitive) representations of $A$ and of $\bar{A}$, respectively. Hence it is easy to see that $T_{\infty}=T_{\infty}^{\prime}$ and $T_{m}=T_{m}^{\prime}$ for every $m$.

Clearly a Banach algebra is radical iff it is topologically nil. It follows that

$$
\operatorname{Rad}^{\prime}(A)=\{a \in A: \rho(a b)=0, \forall b \in \bar{A}\}
$$

for every $A \in(N A)$. So $\operatorname{Rad}^{\prime}(A)$ is a closed topologically nil ideal of $A$, and, for every $A \in(Q A), \operatorname{Rad}^{\prime}(A) \subset \operatorname{rad}(A)$ by Corollary 2.19 .

2.9. Universal envelopes. Now we find out a natural universal class of normed algebras on which $\operatorname{rad}_{b}, \operatorname{rad}_{n}$ and $\operatorname{Rad}^{\prime}$ coincide. The comparison of these radicals on the different classes will be done in the next subsection.

Let $\mathcal{A}$ be a class of normed algebras. Let $\mathcal{A}^{g}$ (resp. $\mathcal{A}^{u}$ ) be the smallest ground (resp. universal) class containing $\mathcal{A}$. We call $\mathcal{A}^{g}$ (resp. $\mathcal{A}^{u}$ ) the ground (resp. universal) envelope of $\mathcal{A}$. Also, let $\mathcal{A}^{i}$ (resp. $\mathcal{A}^{d i}$ ) be the class of all normed algebras topologically isomorphic to ideals (resp. dense ideals) of algebras in $\mathcal{A}$.

Lemma 2.23. Let $\mathcal{A}$ be a ground class. Then so is $\mathcal{A}^{i}$ and $\mathcal{A}^{i}=\mathcal{A}^{\text {di }}$.

Proof. For $\mathcal{A}^{i} \subset \mathcal{A}^{d i}$ it suffices to note that if $A \in \mathcal{A}^{i}$ then $A$ is identified with an ideal of some algebra $B \in \mathcal{A}$. Let $\widetilde{A}$ be the closure of $A$ in $B$. Then $\widetilde{A}$ is a closed ideal of $B$ (hence $\widetilde{A} \in \mathcal{A}$ ) and $A$ is an ideal of $\widetilde{A}$. Therefore $A \in \mathcal{A}^{d i}$. This shows that $\mathcal{A}^{i} \subset \mathcal{A}^{d i}$, and the converse is evident.

Now let $I$ be a closed ideal of $A$. Let $\widetilde{I}$ be the closure of $I$ in $\widetilde{A}$. Then $\widetilde{I}$ is a closed ideal of $\widetilde{A}$ so that $\widetilde{I} \in \mathcal{A}$, and $I=\widetilde{I} \cap A$. Note that $\widetilde{I} A \cup A \widetilde{I} \subset \widetilde{I} \cap A=I$, also

$$
I \widetilde{I}=(\widetilde{I} \cap A) \widetilde{I} \subset \widetilde{I} \cap A \widetilde{I} \subset \widetilde{I} \cap A=I
$$

and, similarly, $\widetilde{I} I \subset I$, i.e., $I$ is an ideal of $\widetilde{I} \in \mathcal{A}$, whence $I \in \mathcal{A}^{i}$.

It remains to show that $A / I \in \mathcal{A}^{i}$. But it is easy: $A / I$ is isometrically isomorphic to a dense ideal of $\widetilde{A} / \widetilde{I} \in \mathcal{A}$, whence $A / I \in \mathcal{A}^{i}$.

Let $\mathcal{A}$ be a class of normed algebras. Put $\mathcal{A}^{(0)}=\mathcal{A}^{g}$ and $\mathcal{A}^{(m+1)}=\left(\mathcal{A}^{(m)}\right)^{i}$ for $m=0,1, \ldots$

TheOREM 2.24. $\mathcal{A}^{u}=\cup_{m \geqslant 0} \mathcal{A}^{(m)}$.

Proof. It is clear that $\mathcal{A}^{u}$ contains $\mathcal{A}^{(0)}=\mathcal{A}^{g}$, and if $\mathcal{A}^{u}$ contains $\mathcal{A}^{(m)}$ then it contains $\mathcal{A}^{(m+1)}$. Therefore $\mathcal{A}^{u} \supset \cup \mathcal{A}^{(m)}$. To the converse it suffices to show that $\cup \mathcal{A}^{(m)}$ is universal. Since every $\mathcal{A}^{(m)}$ is ground then $\cup \mathcal{A}^{(m)}$ is a ground class by Proposition 2.2. Further, if $A \in \cup \mathcal{A}^{(m)}$, say if $A \in \mathcal{A}^{(k)}$ for some $k$, then every ideal of $A$ is contained in $\mathcal{A}^{(k+1)}$. So $\cup \mathcal{A}^{(m)}$ is universal.

THEOREM 2.25. Let $R_{1}$ and $R_{2}$ be HTRs on a universal class $\mathcal{B}$. If $R_{1}=R_{2}$ on a ground class $\mathcal{A} \subset \mathcal{B}$ then $R_{1}=R_{2}$ on $\mathcal{A}^{u}$.

Proof. It is clear that $\mathcal{A}^{u} \subset \mathcal{B}$. Note that $R_{1}=R_{2}$ on $\mathcal{A}^{(0)}$. Suppose that $R_{1}=R_{2}$ on $\mathcal{A}^{(k)}$. If $A \in \mathcal{A}^{(k+1)}$ then $A$ is identified with an ideal of some $B \in \mathcal{A}^{(k)}$. Since $R_{1}$ and 
$R_{2}$ are HTRs on $\mathcal{A}^{u}$, we have

$$
R_{1}(A)=R_{1}(B) \cap A=A \cap R_{2}(B)=R_{2}(A) .
$$

Therefore $R_{1}=R_{2}$ on $\mathcal{A}^{(k+1)}$, hence on $\cup \mathcal{A}^{(m)}=\mathcal{A}^{u}$.

Corollary 2.26. $\operatorname{Rad}^{\prime}=\operatorname{rad}_{n}=\operatorname{rad}_{b}=\operatorname{rad}$ on $(B A)^{u}$.

Proof. Recall that these radicals are HTRs on $(Q A)$ and clearly $(B A)^{u} \subset(Q A)$. Apply Theorem 2.25.

Note that $(B A)^{u} \subset\left(Q_{b} A\right)$ because $\left(Q_{b} A\right)$ is a universal class containing $(B A)$. We will show in the next subsection that the inclusion is strict.

2.10. Comparison of the radicals. Let us write $R_{1} \leqslant R_{2}$ on a class $\mathcal{A}$ if $R_{1}(A) \subset R_{2}(A)$, for each $A \in \mathcal{A}$; if, for some $A \in \mathcal{A}$, the inclusion is strict we write $R_{1}<R_{2}$ on $\mathcal{A}$.

It is clear that $\operatorname{rad}_{n} \leqslant \operatorname{rad}_{b}$ on $(N A)$; we will prove that they differ already on $(Q A)$.

Proposition 2.27. The inclusion $\left(Q_{b} A\right) \subset(Q A)$ is strict and $\operatorname{rad}_{n}<\operatorname{rad}_{b}$ on $(Q A)$.

Proof. It will be sufficient to construct an algebra $A \in(Q A) \backslash\left(Q_{b} A\right)$ with $\operatorname{rad}_{n}(A) \neq$ $\operatorname{rad}_{b}(A)$.

Let $H$ be a separable Hilbert space. Given a basis we identify an operator on $H$ with a matrix. Let $A$ be the algebra of all matrices with only a finite number of nonzero entries, equipped with the operator norm. Every element of $A$ generates a finite-dimensional subalgebra, whence $\sum_{n>0} a^{n}$ converges, for $\|a\|<1$. So $A$ is a $Q$-algebra.

Note that $A$ has no nonzero proper ideals. Indeed, if $I$ is such an ideal, then $I$ contains an operator with only one nonzero entry and therefore all operators in $A$, i.e. coincides with $A$, a contradiction.

Clearly $A$ is the union of a sequence of finite-dimensional subalgebras $A_{n}$. So, for a nonzero representation $\pi$ of $A$ on a Banach space $X, \pi(A) x$ has a countable Hamel basis for every vector $x \in X$ and cannot coincide with $X$ if $\operatorname{dim} X=\infty$. If $\operatorname{dim} X<\infty$ then ker $\pi$ is a nonzero proper ideal of $A$, that is impossible. Hence $A$ has no strictly irreducible representations on Banach spaces, whence $A=\operatorname{rad}_{b}(A)$.

On the other hand, the linear span $H_{0}$ of the basis is invariant for $A$ and the restriction of $A$ to $H_{0}$ is a strictly irreducible representation. Hence $A \notin\left(Q_{b} A\right)$ and $\operatorname{rad}_{n}(A)=0$.

Proposition 2.28. $\operatorname{Rad}^{\prime}<\operatorname{rad}_{n}$ on $(N A) ;$ moreover, $\operatorname{Rad}^{\prime}<\operatorname{rad}$ on $\left(Q_{b} A\right)$ and the inclusion $(B A)^{u} \subset\left(Q_{b} A\right)$ is strict.

Proof. Since $\operatorname{Rad}^{\prime}(A)$ is a topologically nil ideal of a normed algebra $A, \operatorname{Rad}^{\prime} \leqslant \operatorname{rad}_{n}$ on $(N A)$ by Theorem $2.17($ ii).

Now we show that $\operatorname{Rad}^{\prime}<\operatorname{rad}$ on $\left(Q_{b} A\right)$. Dixon [7, Example 9.3] constructed a radical Banach algebra $A$ and a continuous isomorphism $\phi: A \rightarrow C$ onto a dense subalgebra $C$ of a semisimple Banach algebra $B$. Then $C$ is radical $(C=\operatorname{rad}(C))$, hence is a $Q_{b}$-algebra. On the other hand, $\operatorname{Rad}^{\prime}(C)=C \cap \operatorname{Rad}(A)=0$.

We see that the inclusion $(B A)^{u} \subset\left(Q_{b} A\right)$ is strict because $\operatorname{Rad}^{\prime}=\operatorname{rad}$ on $(B A)^{u}$ by Corollary 2.26 .

This indicates that $R^{\prime}$ need not be a maximal extension of an HTR $R$, and, as a consequence, an HTR on $(N A)$ need not be semi-regular. 
Proposition 2.29. $T_{\infty}<\operatorname{Rad}^{\prime}$ on $(N A)$.

Proof. Clearly $T_{\infty} \leqslant \operatorname{Rad}$ on $(B A)$ because strictly irreducible representations of Banach algebras are strictly dense; the inequality is strict by [7, Theorem 9.2 and example 9.3]. By regularity, $T_{\infty}<\operatorname{Rad}^{\prime}$ on $(N A)$.

As a consequence of the results, we have strict inclusions in the following chain of universal classes:

$$
(B A)^{u} \subset\left(Q_{b} A\right) \subset(Q A)
$$

Let us also write together the strict inequalities obtained for radicals on $(N A)$ :

$$
T_{\infty}<\operatorname{Rad}^{\prime}<\operatorname{rad}_{n}<\operatorname{rad}_{b}
$$

2.11. Topologically characteristic and symmetric radicals. The following notion can be of use in dealing with Lie subalgebras of normed algebras.

A TR $R$ is called topologically characteristic on a class $\mathcal{A}$ if, for every $A \in \mathcal{A}$ and every bounded derivation $D$ of $A, D R(A) \subset R(A)$.

LEMma 2.30. All (BA)-radicals are topologically characteristic.

Proof. Let $R$ be a TR on $(B A)$. If $D$ is a bounded derivation on a Banach algebra $A$ then $\exp (\lambda D)$ is an automorphism of $A$ for every $\lambda \in \mathbb{C}$, whence

$$
\exp (\lambda D) R(A)=R(A) \text {. }
$$

Since $R(A)$ is closed in $A$,

$$
\lim _{\lambda \rightarrow 0}(\exp (\lambda D) a-a) / \lambda \in R(A)
$$

for every $a \in R(A)$, whence $D(a) \in R(A)$.

Theorem 2.31. Let $\mathcal{A}$ be a class such that $(B A) \subset \mathcal{A}$. Any regular $T R R$ on $\mathcal{A}$ is topologically characteristic.

Proof. Indeed, for every bounded derivation $D$ on an algebra $A \in \mathcal{A}$, we have

$$
D R(A)=D(A \cap R(\bar{A})) \subset D A \cap D R(\bar{A}) \subset A \cap R(\bar{A})=R(A) .
$$

In particular $\operatorname{Rad}^{\prime}, T_{\infty}$ and $T_{m}$ are topologically characteristic on $(N A)$, for every $m$. TheOREM 2.32. $\operatorname{rad}_{b}$ and $\operatorname{rad}_{n}$ are topologically characteristic on $(N A)$.

Proof. Let $D$ be a bounded derivation on an algebra $A \in(N A)$, and let $a \in \operatorname{rad}_{b}(A)(\operatorname{resp}$. $\left.\operatorname{rad}_{n}(A)\right)$ be arbitrary. By [21, Lemma 2.1], $\pi(D a)$ is quasinilpotent for every $\pi \in \operatorname{irr}_{b} A$ (resp. $\operatorname{irr}_{n} A$ ). If $y=\pi(D a) x \neq 0$ for some $\pi$ and $x \in X_{\pi}$, then there is an element $b \in A$ such that $\pi(b) y=x$, Hence

$$
\pi(D(a b)) y=\pi(D a) \pi(b) y+\pi(a) \pi(D b) y=\pi(D a) x=y
$$

and also $a b \in \operatorname{rad}_{b}(A)$ (resp. $\operatorname{rad}_{n}(A)$ ), a contradiction. Therefore $\operatorname{rad}_{b}$ and $\operatorname{rad}_{n}$ are topologically characteristic on $(N A)$.

Many of the TRs considered were defined in an asymmetric way: by using representations. For instance the 'left-defined' TRs are $\operatorname{rad}_{n}, \operatorname{rad}_{b}, T_{\infty}$ etc. On the other hand, using anti-representations for definition of similar, 'right-defined' radicals is also a right way. So, we consider the opposite TRs for asymmetric TRs in the general setting. 
Let $A^{\text {op }}$ be the same algebra $A$, but with the opposite multiplication. Recall that $A^{\text {op }}$ is called an opposite algebra. The 'identity' anti-isomorphism $a \mapsto a^{\text {op }}$ maps an element $a$ to the same element but in $A^{\mathrm{op}}$; note that $a^{\mathrm{op}} b^{\mathrm{op}}=(b a)^{\mathrm{op}}$ for all $a, b \in A$ and every homomorphism $f: A \rightarrow B$ induces the opposite homomorphism $f^{\text {op }}: A^{\text {op }} \rightarrow B^{\text {op }}$ by formula $f^{\mathrm{op}}\left(a^{\mathrm{op}}\right)=(f(a))^{\mathrm{op}}$, for every $a \in A$. Clearly $\left(f^{\mathrm{op}}\right)^{\mathrm{op}}=f$.

Let $\mathcal{A} \subset(N A)$, and let $\mathcal{A}^{\text {op }}$ be the class of all algebras $A$ such that $A^{\text {op }} \in \mathcal{A}$. Clearly $\left(\mathcal{A}^{\mathrm{op}}\right)^{\mathrm{op}}=\mathcal{A}$; we call $\mathcal{A}^{\mathrm{op}}$ an opposite class. All properties considered above are inherited by the opposite class. A class $\mathcal{A}$ is called symmetric if $\mathcal{A}=\mathcal{A}^{\mathrm{op}}$. Note that $(B A)$ and $(Q A)$ are symmetric. Moreover, all closed subalgebras of a $Q$-algebra are $Q$-algebras. This is not clear for $Q_{b}$-algebras. Is $\left(Q_{b} A\right)$ symmetric? Does it contain all closed subalgebras of its algebras?

Let $\mathcal{A}$ be a ground class. Then clearly $\mathcal{A}^{\mathrm{op}}$ is a ground class. If $R$ is defined on $\mathcal{A}$, one can define $R^{\text {op }}$ on $\mathcal{A}^{\text {op }}$ by

$$
R^{\mathrm{op}}\left(A^{\mathrm{op}}\right)=R(A)^{\mathrm{op}}
$$

for every $A \in \mathcal{A}$. We call $R^{\mathrm{op}}$ opposite to $R$. If $\mathcal{A}$ is symmetric, $R$ is called symmetric on $\mathcal{A}$ if $R(A)=R^{\mathrm{op}}(A)$ for every $A \in \mathcal{A}$.

Proposition 2.33. Let $R$ be a TR or HTR on a ground class $\mathcal{A}$. Then so is $R^{\text {op }}$ on $\mathcal{A}^{\mathrm{op}}$.

Proof. For $\left(1^{\circ}\right)$, we have

$$
R^{\mathrm{op}}\left(A^{\mathrm{op}}\right)=R(A)^{\mathrm{op}}=R(R(A))^{\mathrm{op}}=R^{\mathrm{op}}\left(R(A)^{\mathrm{op}}\right)=R^{\mathrm{op}}\left(R^{\mathrm{op}}\left(A^{\mathrm{op}}\right)\right) .
$$

The easy checkup of the other properties is left to the reader.

Note that $\operatorname{Rad}^{\prime}$ is symmetric on $(N A)$ and $\operatorname{rad}_{n}$ on $(Q A)$. Are $\operatorname{rad}_{n}$ and $\operatorname{rad}_{b}$ symmetric on $(N A)$ ?

3. Tensor radicals. In this section we consider the behavior of a topological radical with respect to the fundamental operations: direct sum $\oplus$ and projective tensor product $\widehat{\otimes}$ of algebras.

3.1. Radicals on direct sums. For direct sums the problem is easy.

Theorem 3.1. Let $\mathcal{A}$ be a ground class. If $R$ is a TR on $\mathcal{A}$ and $A \oplus B \in \mathcal{A}$ for some $A, B \in \mathcal{A}$ then $R(A \oplus B)=R(A) \oplus R(B)$.

Proof. Let $f_{1}$ and $f_{2}$ be the epimorphisms of $A \oplus B$ onto $A$ and $B$ respectively, defined as the natural projections (which are open and continuous). Then, by $\left(4_{f}^{\circ}\right)$, projections of $R(A \oplus B)$ are contained in $R(A)$ and $R(B)$, respectively. Hence

$$
R(A \oplus B) \subset R(A) \oplus R(B) .
$$

On the other hand, if one considers the ideals $I_{1}=A \oplus 0$ and $I_{2}=0 \oplus B$ and applies $\left(5^{\circ}\right)$ then the inclusions $R(A \oplus 0) \subset R(A \oplus B)$ and $R(0 \oplus B) \subset R(A \oplus B)$ will be established. But $f_{1}$ defines a topological isomorphism of $A \oplus 0$ onto $A$, whence $\left(3^{\circ}\right)$ gives $R(A \oplus 0)=$ $R(A) \oplus 0$ and, similarly, $R(0 \oplus B)=0 \oplus R(B)$. So

$$
R(A) \oplus R(B) \subset R(A \oplus B)
$$

and we are done. 
3.2. Tensor and weakly tensor radicals on $(B A)$. The second question is much more difficult. We will consider it only for topological radicals on $(B A)$ because the projective tensor product is a Banach space operation, i.e. for $A$ and $\bar{A}$ the result is the same.

Let us denote by $A \otimes B$ the algebraic tensor product of Banach algebras $A$ and $B$. Clearly $A \otimes B$ can be considered as a linear manifold of $A \widehat{\otimes} B$. For all subsets $M \subset A$ and $N \subset B$ that are not linear manifolds, it is convenient to denote by $M \otimes N$ the set $\{a \otimes b \in A \otimes B: a \in M, b \in N\}$. If one of them is a linear manifold, let $M \otimes N$ be the linear span in $A \otimes B$ of all elements $a \otimes b, a \in M, b \in N$. In any case, $M \otimes N$ can be also considered as a subset of $A \widehat{\otimes} B$. Let $\widehat{M \otimes N}$ denote the closure of $M \otimes N$ in $A \widehat{\otimes} B$.

A topological radical $R$ on $(B A)$ is called tensor if

$$
R(A) \otimes B \subset R(A \widehat{\otimes} B),
$$

for any Banach algebras $A, B$. For a wide class of TRs this condition admits a convenient reformulation.

TheOREM 3.2. An ideally strong radical $R$ on $(B A)$ is tensor if and only if the tensor product of an $R$-radical algebra and arbitrary Banach algebra is $R$-radical.

The proof will be given after some preliminary work.

If $J$ is a closed ideal in a Banach algebra $A$ then, for any Banach algebra $B$, the 'identity' map $i_{A}: J \widehat{\otimes} B \rightarrow A \widehat{\otimes} B$ is contracting. The following lemma is probably known, but we could not find a precise reference.

Lemma 3.3. Let $A, B \in(B A)$, and let $J$ be a closed ideal of $A$.

(i) $i_{A}(J \widehat{\otimes} B)$ is a two-sided ideal (non-necessarily closed) of $A \widehat{\otimes} B$ consisting of all elements that can be represented in the form $\sum a_{n} \otimes b_{n}$ with $a_{n} \in J$ and

$$
\sum\left\|a_{n}\right\|\left\|b_{n}\right\|<\infty \text {. }
$$

(ii) There exists a unique contractive epimorphism $\tau_{J}:(A / J) \widehat{\otimes} B \rightarrow(A \widehat{\otimes} B) / U$, where $U$ is the closure of $i_{A}(J \widehat{\otimes} B)$ in $A \widehat{\otimes} B$, such that

$$
\tau_{J}((a+J) \otimes b)=a \otimes b+U
$$

for every $a \in A, b \in B$.

Proof. (i) is evident.

(ii) Let $E=A \widehat{\otimes} B / U$ and, as usual, $q_{U}: A \widehat{\otimes} B \rightarrow E$ the canonical epimorphism. We define a linear map $\tau:(A / J) \otimes B \rightarrow E$ by

$$
\tau\left(\sum\left(a_{n}+J\right) \otimes b_{n}\right)=q_{U}\left(\sum a_{n} \otimes b_{n}\right)
$$

To justify the definition, note first that it does not depend on the choice of representatives: if $a_{n}+J=a_{n}^{\prime}+J$ for every $n$ then $\sum a_{n} \otimes b_{n}-\sum a_{n}^{\prime} \otimes b_{n} \in J \otimes B$ and therefore

$$
q_{U}\left(\sum a_{n} \otimes b_{n}\right)=q_{U}\left(\sum a_{n}^{\prime} \otimes b_{n}\right)
$$

So it suffices to check the bilinearity which is easy.

Now we have to prove that $\tau$ is contractive. For any $T \in(A / J) \otimes B$ and any $\varepsilon>0$, there exist $a_{n} \in A, b_{n} \in B(n=1, \ldots, m)$ such that $T=\sum\left(a_{n}+J\right) \otimes b_{n},\left\|b_{n}\right\|=1$ and

$$
\sum\left\|a_{n}+J\right\|<\|T\|+\varepsilon .
$$


Choosing $a_{n}^{\prime} \in a_{n}+J$ with

$$
\left\|a_{n}^{\prime}\right\|<\left\|a_{n}+J\right\|+\varepsilon / m
$$

we get that

$$
\begin{aligned}
\|\tau(T)\| & =\left\|q_{U}\left(\sum a_{n}^{\prime} \otimes b_{n}\right)\right\| \leqslant\left\|\sum a_{n}^{\prime} \otimes b_{n}\right\| \leqslant\left\|\sum a_{n}^{\prime}\right\| \\
& <\|T\|+2 \varepsilon .
\end{aligned}
$$

Since $\varepsilon$ is arbitrary, $\|\tau(T)\| \leqslant\|T\|$. The linearity and multiplicativity of $\tau$ are evident.

Denote by $\tau_{J}$ the contractive homomorphism of $(A / J) \widehat{\otimes} B$ to $E$ that extends $\tau$ by continuity. Then

$$
\tau_{J}\left(\sum_{1}^{\infty}\left(a_{n}+J\right) \otimes b_{n}\right)=q_{U}\left(\sum_{1}^{\infty} a_{n} \otimes b_{n}\right)
$$

whenever $\sum\left\|a_{n}\right\|\left\|b_{n}\right\|<\infty$. Hence $\tau_{J}$ is surjective.

LEMma 3.4. A topological radical $R$ on $(B A)$ is tensor iff

$$
i_{A}(R(A) \widehat{\otimes} B) \subset R(A \widehat{\otimes} B),
$$

for all Banach algebras $A, B$.

Proof. Follows from the evident inclusions

$$
R(A) \otimes B \subset i_{A}(R(A) \widehat{\otimes} B) \subset R \widetilde{R(A) \otimes B}
$$

(the latter is, as usual, the closure of $R(A) \otimes B$ in $A \widehat{\otimes} B$ ) and the fact that $R(A \widehat{\otimes} B)$ is closed.

Proof of Theorem 3.2. If $R$ is tensor and $A$ is $R$-radical then

$$
A \otimes B=R(A) \otimes B \subset R(A \widehat{\otimes} B),
$$

whence $A \widehat{\otimes} B \subset R(A \widehat{\otimes} B)$ and accordingly $A \widehat{\otimes} B$ is $R$-radical.

Conversely, let $R$ be ideally strong and suppose that the tensor product of an $R$-radical algebra and arbitrary Banach algebra is $R$-radical. Let $A, B \in(B A)$ be arbitrary. The map $i_{A}: R(A) \widehat{\otimes} B \rightarrow A \widehat{\otimes} B$ is a continuous epimorphism onto the ideal $i_{A}(R(A) \widehat{\otimes} B)$ of $A \widehat{\otimes} B$ (see Lemma 3.3) and the algebra $R(A) \widehat{\otimes} B$ is $R$-radical by our assumptions. Hence

$$
i_{A}(R(A) \widehat{\otimes} B)=i_{A}(R(R(A) \widehat{\otimes} B)) \subset R(A \widehat{\otimes} B) .
$$

Using Lemma 3.4, we conclude that $R$ is tensor.

We will say that a $(B A)$-radical $R$ is weakly tensor if $A \widehat{\otimes} B$ is $R$-radical, for any $R$ radical algebra $A$ and arbitrary algebra $B$. So Theorem 3.2 states that a weakly tensor, ideally strong radical is tensor.

Recall that the class $(B A)^{i}$ of all normed algebras topologically isomorphic to ideals of Banach algebras is a ground class (Lemma 2.23) and that all $(B A)$-radicals are strong. The following simple assertion underlines the important role of existence of strong extensions of weakly tensor $(B A)$-radicals to $(B A)^{i}$.

Proposition 3.5. Let $R$ be a weakly tensor $T R$ on $(B A)$. If $R$ admits an extension to $(B A)^{i}$ as a strong $T R$ then $R$ is tensor. 
Proof. Indeed, in this case $R$ is ideally strong and hence tensor by Theorem 3.2 .

Some examples of tensor or weakly tensor radicals will be given in this and the next sections as well as in subsequent publications of our project.

3.3. The radical $R^{t}$. In general, given a TR $R$ on $(B A)$, one can try to construct a related tensor radical $R^{t}$ in the following way:

$$
R^{t}(A)=\{a \in A: a \otimes B \subset R(A \widehat{\otimes} B), \quad \forall B \in(B A)\} .
$$

It follows from the above arguments that if $R^{t}$ is a TR then it is a tensor one (because of the associativity of tensor product) and that $R$ itself is tensor iff $R=R^{t}$. It is important to know conditions under which $R^{t}$ is a TR. We will obtain now some results in this direction.

Let $I$ be a closed ideal of a Banach algebra $A$. For a Banach algebra $B$, the homomorphism $i_{A}: I \widehat{\otimes} B \rightarrow A \widehat{\otimes} B$ is injective if, say, $I$ has a bounded approximate identity. Moreover, in this case $i_{A}$ is bounded from below. Indeed, given a b.a.i. $e_{\lambda}$ in $I$ with $\beta=\sup \left\|e_{\lambda}\right\|$, define a net of operators $S_{\lambda}$ on $I \widehat{\otimes} B$ by $S_{\lambda}(a \otimes b)=e_{\lambda} a \otimes b$. Then $S_{\lambda} \rightarrow 1$ in the strong operator topology. Now if $G \in I \widehat{\otimes} B$ and $F=i_{A}(G)$ then, for any presentation of $F$ in the form $F=\sum a_{n} \otimes b_{n}$, one has

$$
\left\|S_{\lambda} G\right\| \leqslant \sum\left\|e_{\lambda} a_{n}\right\|\left\|b_{n}\right\| \leqslant \beta \sum\left\|a_{n}\right\|\left\|b_{n}\right\| \text {. }
$$

It follows that $\left\|S_{\lambda} G\right\| \leqslant \beta\|F\|$ and, passing to the limit, $\|G\| \leqslant \beta\|F\|$.

In general $i_{A}$ can have a non-zero kernel $K=K(A, I, B)$. The algebras $K(A, I, B)$ will be called tensor pathological algebras.

ThEOREM 3.6. Let $R$ be an HTR on $(B A)$. Then

(i) $R^{t}(A)$ is a closed ideal of $A$, for any Banach algebra $A$.

(ii) If $R$ is ideally strong then $R^{t}$ satisfies conditions $\left(2^{\circ}\right),\left(3^{\circ}\right),\left(4^{\circ}\right)$ and $\left(5_{2}^{\circ}\right)$.

(iii) If $R$ is strictly hereditary and if all tensor pathological algebras are $R$-radical then $R^{t}$ is an $H T R$ on $(B A)$.

Proof. (i) Let $I=\{a \in A: a \otimes B \subset R(A \widehat{\otimes} B)$ for every unital $B \in(B A)\}$. Then $I$ is an ideal of $A$ : if $a \otimes b \in R(A \widehat{\otimes} B)$ then

$$
(c a) \otimes b=(c \otimes 1)(a \otimes b) \in R(A \otimes B) .
$$

Clearly $R^{t}(A) \subset I$. We will prove that $I=R^{t}(A)$.

Note first that if $B$ is a non-unital Banach algebra and $j$ the natural homomorphism of $A \widehat{\otimes} B$ to $A \widehat{\otimes} B^{1}$ then $j$ is isometric and $j(A \widehat{\otimes} B)$ is a closed ideal of $A \widehat{\otimes} B^{1}$ (here $A \widehat{\otimes} B^{1}$ is the product of $A$ and $\left.B^{1}\right)$.

Indeed, if $F \in A \widehat{\otimes} B$ and $j(F)$ has a representative $\sum a_{n} \otimes\left(b_{n}+\lambda_{n}\right)$ with $\lambda_{n}$ scalar multiplies of the unit such that, given $\varepsilon>0$,

$$
\|j(F)\|+\varepsilon>\sum\left\|a_{n}\right\|\left\|b_{n}+\lambda_{n}\right\|=\sum\left\|a_{n}\right\|\left(\left\|b_{n}\right\|+\left|\lambda_{n}\right|\right)
$$

(using the standard norm in $B \oplus \mathbb{C}=B^{1}$ ) then $\sum \lambda_{n} a_{n}$ converges to 0 since $A \widehat{\otimes} B$ is a complemented subspace of $A \widehat{\otimes} B^{1}$, whence $F=\sum a_{n} \otimes b_{n}$ and

$$
\|j(F)\|+\varepsilon>\sum\left\|a_{n}\right\|\left\|b_{n}\right\| \geqslant\|F\| \text {. }
$$


It follows that $\|j(F)\| \geqslant\|F\|$. The converse inequality is evident. Hence clearly $j(A \widehat{\otimes} B)$ is a closed ideal of $A \widehat{\otimes} B^{1}$.

If $a \in I$ then $a \otimes B^{1} \subset R\left(A \widehat{\otimes} B^{1}\right)$,

$$
j(a \otimes B) \subset j(A \widehat{\otimes} B) \cap R\left(A \widehat{\otimes} B^{1}\right)=R(j(A \widehat{\otimes} B))=j(R(A \widehat{\otimes} B))
$$

(we used $\left(6^{\circ}\right)$ and $\left(3^{\circ}\right)$ for $R$ ), whence $a \otimes B \subset R(A \widehat{\otimes} B)$ and $a \in R^{t}(A)$. Thus $I=R^{t}(A)$ is an ideal of $A$, obviously closed.

(ii) Let $I$ be a closed ideal of a Banach algebra $A$. If $a \in R^{t}(I)$ then, for each $B \in$ ( $B A)$ and each $b \in B, a \otimes b \in R(I \widehat{\otimes} B)$. By Lemma 3.3, the image of the continuous homomorphism $i_{A}: I \widehat{\otimes} B \rightarrow A \widehat{\otimes} B$ is an ideal of $A \widehat{\otimes} B$. Since $R$ is ideally strong, $i_{A}(a \otimes b) \in$ $R(A \widehat{\otimes} B)$, that is, $a \otimes b \in R(A \widehat{\otimes} B)$. This means that $a \in R^{t}(A)$, whence $R^{t}(I) \subset I \cap R^{t}(A)$. We proved that $R^{t}$ satisfies $\left(5_{2}^{\circ}\right)$.

Now let us show that $q_{I}\left(R^{t}(A)\right) \subset R^{t}(A / I)$. Let $a \in R^{t}(A)$. Then $a \otimes b \in R(A \widehat{\otimes} B)$ for each $B \in(B A)$. Let $f: A \widehat{\otimes} B \rightarrow(A / I) \widehat{\otimes} B$ be the epimorphism defined by $f(x \otimes y)=$ $(x+I) \otimes y$ for every $x \in A, y \in B$. Then

$$
f(a \otimes b) \in R((A / I) \widehat{\otimes} B), \quad(a+I) \otimes b \in R((A / I) \widehat{\otimes} B),
$$

whence $a+I \in R^{t}(A / I)$, and therefore $R^{t}$ satisfies $\left(4^{\circ}\right)$.

The property $\left(3^{\circ}\right)$ is evident and we have to prove $\left(2^{\circ}\right)$. Denote $R^{t}(A)$ by $J$ for brevity. Let, as in Lemma $3.3, U$ be the closure of $i_{A}(J \widehat{\otimes} B)$ in $A \widehat{\otimes} B$ and $\tau_{J}$ the epimorphism of $(A / J) \widehat{\otimes} B$ onto $(A \widehat{\otimes} B) / U$. Since $i_{A}(J \widehat{\otimes} B)$ is an ideal of $A \widehat{\otimes} B$ (Lemma 3.3) and $R$ is ideally strong, the ideal $U$ is $R$-radical. Using Corollary 2.8(ii), we get that

$$
R((A \widehat{\otimes} B) / U)=q_{U}(R(A \widehat{\otimes} B)) .
$$

Furthermore, it follows from $\left(7^{\circ}\right)$ (that holds for $R$ ) that

$$
\tau_{J}(R((A / J) \widehat{\otimes} B)) \subset R((A \widehat{\otimes} B) / U) .
$$

Therefore, it follows from (3.2) and (3.3) that

$$
\tau_{J}(R((A / J) \widehat{\otimes} B)) \subset q_{U}(R(A \widehat{\otimes} B)) .
$$

Let now $a+J \in R^{t}(A / J)$. Then $(a+J) \otimes b \subset R((A / J) \widehat{\otimes} B)$, whence

$$
\tau_{J}((a+J) \otimes b) \in q_{U}(R(A \widehat{\otimes} B)),
$$

and $a \otimes b+U \in q_{U}(R(A \widehat{\otimes} B))$. Since $U \subset R(A \widehat{\otimes} B)$, we conclude that $a \otimes b \in R(A \widehat{\otimes} B)$. Hence $a \in R^{t}(A)=J$ and $a+J=0$. This shows that $\left(2^{\circ}\right)$ holds for $R^{t}$.

(iii) Suppose now that $R$ is strictly hereditary and all tensor pathological algebras are $R$-radical. We need only prove the property $\left(6^{\circ}\right)$ (which implies $\left(1^{\circ}\right)$ and $\left(5_{1}^{\circ}\right)$ ). Let again $I$ be a closed ideal of a Banach algebra $A$. Fix $B \in(B A)$ and denote by $K$ the kernel of the homomorphism $i_{A}: I \widehat{\otimes} B \rightarrow A \widehat{\otimes} B$ and by $h$ the induced homomorphism of $(I \widehat{\otimes} B) / K$ to $A \widehat{\otimes} B$. Note that $i_{A}=h q_{K}$. It is important that $h$ is injective and its image is an ideal of $A \widehat{\otimes} B$.

Let now $a \in I \cap R^{t}(A)$. Then, for each $b \in B$, the element $i_{A}(a \otimes b)$ belongs to $R(A \widehat{\otimes} B)$ (here we consider $a \otimes b$ as an element of $I \widehat{\otimes} B$ ). Since $R$ is strictly hereditary,

$$
h(R((I \widehat{\otimes} B) / K))=i_{A}(I \widehat{\otimes} B) \cap R(A \widehat{\otimes} B),
$$


whence

$$
i_{A}(a \otimes b) \in h(R((I \widehat{\otimes} B) / K)) .
$$

Since the algebra $K$ is tensor pathological, it is $R$-radical and, by Corollary 2.8(ii),

$$
R((I \widehat{\otimes} B) / K)=q_{K}(R(I \widehat{\otimes} B)) .
$$

Thus

$$
i_{A}(a \otimes b) \in h\left(q_{K}(R(I \widehat{\otimes} B))\right)=i_{A}(R(I \widehat{\otimes} B)),
$$

whence

$$
a \otimes b-F \in \operatorname{ker} i_{A}
$$

for some $F \in R(I \widehat{\otimes} B)$. Since $\operatorname{ker} i_{A}=K$ is $R$-radical, it is contained in $R(I \widehat{\otimes} B)$, that gives

$$
a \otimes b \in R(I \widehat{\otimes} B) .
$$

Therefore $a \in R^{t}(I)$. We proved the inclusion $I \cap R^{t}(A) \subset R^{t}(I)$. The converse inclusion was established from $\left(5_{2}^{\circ}\right)$.

3.4. Tensor properties of the Jacobson radical. We know that the Jacobson radical Rad is strictly hereditary (see Corollary 2.15). But the result of Theorem 3.6 cannot be immediately applied to $R=$ Rad because we do not know if the tensor pathological algebras are radical. The proof of the fact that $\operatorname{Rad}^{t}$ is an HTR will be finished by means of a technique related to the notion of joint quasinilpotence.

The most interesting and important problem is one of the coincidence of $\operatorname{Rad}^{t}$ and Rad. In the form "is the projective tensor product of a radical Banach algebra $A$ and arbitrary Banach algebra $B$ radical?" it in fact goes back to [1], where the case of commutative $B$ was solved. Being unable to solve it in full generality, we consider its particular aspects.

Let us call a Banach algebra $A$ tensor radical if $A=\operatorname{Rad}^{t}(A)$ that is if $A \widehat{\otimes} B$ is the Jacobson radical for arbitrary Banach algebra $B$; $A$ is called tensor perfect if $A \widehat{\otimes} B$ is radical, for any radical Banach algebra $B$.

In what follows it is convenient to deal with 'generalized subsets' of a normed algebra.

Let $G$ be an arbitrary set, $M=\left(a_{\alpha}\right)_{\alpha \in \Lambda}$ and $N=\left(b_{\beta}\right)_{\beta \in \Omega}$ families of elements of $G$. We write $M \subset N$ if there exists an one-to-one map $\varphi$ from $\Lambda$ in $\Omega$ such that $b_{\varphi(\alpha)}=a_{\alpha}$ for every $\alpha \in \Lambda$, and $M \simeq N$ if $M \subset N$ and $N \subset M$. The relation $\simeq$ is clearly an equivalence relation on the set of all families of elements of $G$. We call the classes of equivalence by generalized subsets of $G$. For brevity we sometimes do not distinguish between a generalized subset and its arbitrary representative.

It is useful to interpret generalized subsets of $G$ as follows. If $M$ is a generalized subset of $G$ with a representative $\left(a_{\alpha}\right)_{\alpha \in \Lambda}$ then one can clearly identify $M$ with the set $M^{\sharp}$ of all pairs $(a, t)$, where $a \in A$ and $t=\operatorname{card}\left\{\alpha \in \Lambda: a_{\alpha}=a\right\}>0$. Considering all such pairs $(a, t)$ for $t \geqslant 0$, we also identify $M$ with the cardinal-valued function $\varkappa_{M}: a \mapsto t$ defined on $G$. We call $\varkappa_{M}$ the functional representation of $M$. It is clear that one can regard usual subsets as generalized ones: their functional representations coincide with their indicators. This justifies the term 'generalized subset'. 
In terms of the functional representations the inclusion $M \subset N$ for generalized subsets of $G$ turns into the inequality

$$
\varkappa_{M}(a) \leqslant \varkappa_{N}(a)
$$

for every $a \in G$. It is convenient to define the union $M \cup N$ and the intersection $M \cap N$ via their functional representations as follows:

$$
\varkappa_{M \cup N}(a)=\max \left\{\varkappa_{M}(a), \varkappa_{N}(a)\right\}
$$

and

$$
\varkappa_{M \cap N}(a)=\min \left\{\varkappa_{M}(a), \varkappa_{N}(a)\right\}
$$

for every $a \in G$. The union and intersection of a collection of generalized subsets are defined similarly.

Now let $A$ be a normed algebra, and let $M, N$ be generalized subsets of $A$. Let $\left(a_{\alpha}\right)_{\alpha \in \Lambda}$ and $\left(b_{\beta}\right)_{\beta \in \Omega}$ be representatives of $M$ and $N$, respectively. Then we define $M N$ as a generalized subset of $A$ with the representative $\left(a_{\alpha} b_{\beta}\right)_{(\alpha, \beta) \in \Lambda \times \Omega}$. Note that

$$
\varkappa_{M N}(a)=\sum_{(b, c) \in A \times A, b c=a} \varkappa_{M}(b) \varkappa_{N}(c)
$$

for every $a \in A$.

Given a generalized subset $M$ of $A$, set

$$
\|M\|_{1}=\sum_{\alpha \in \Lambda}\left\|a_{\alpha}\right\|
$$

(the sum is calculated as $\sup _{\Delta \subset \Lambda} \sum_{\alpha \in \Delta}\left\|a_{\alpha}\right\|$, where $\Delta$ runs over all finite subsets of $\Lambda$ ). Clearly $\|M\|_{1}$ does not depend on the choice of a representative of $M$. If $\|M\|_{1}<\infty$, we say that $M$ is summable. Note that if $M$ is summable then the set $M^{\sharp}$ is (finite or) countable, $\varkappa_{M}(a)<\infty$ for every $a \in A$ and

$$
\|M\|_{1}=\sum_{a \in A} \varkappa_{M}(a)\|a\| .
$$

Furthermore, clearly

$$
\|M N\|_{1} \leqslant\|M\|_{1}\|N\|_{1}
$$

whence, setting $M^{n}=M M \cdots M$ ( $n$ times $)$,

$$
\left\|M^{n+m}\right\|_{1} \leqslant\left\|M^{n}\right\|_{1}\left\|M^{m}\right\|_{1}
$$

for every $n, m \in \mathbb{N}$. It follows from (3.6) that, for every summable generalized subset $M$ of $A$, there exists a limit

$$
\rho_{1}(M)=\lim \left(\left\|M^{n}\right\|_{1}\right)^{1 / n}=\inf \left(\left\|M^{n}\right\|_{1}\right)^{1 / n} .
$$

Note that $\left(M^{m}\right)^{n}=M^{m n}$ for every $n, m \in \mathbb{N}$, whence

$$
\rho_{1}\left(M^{m}\right)^{1 / m}=\left(\lim _{n}\left(\left\|\left(M^{m}\right)^{n}\right\|_{1}\right)^{1 / n}\right)^{1 / m}=\lim _{n}\left(\left\|M^{m n}\right\|_{1}\right)^{1 / n m}=\rho_{1}(M) .
$$

If $f$ is a map from $A$ into a normed algebra $B, f M$ denotes the generalized subset of $B$ with the representative $\left(f a_{\alpha}\right)_{\alpha \in \Lambda}$; in particular, if $I$ is a closed ideal of $A$ then $M / I$ denotes the generalized subset of $A / I$ with the representative $\left(a_{\alpha}+I\right)_{\alpha \in \Lambda}$. Note that

$$
\varkappa_{f M}(b)=\sum_{a \in A, f a=b} \varkappa_{M}(a)
$$


for every $b \in B$. If $f$ is a bounded homomorphism then

$$
\rho_{1}(f M)=\lim \left(\left\|(f M)^{n}\right\|_{1}\right)^{1 / n} \leqslant \lim \|f\|^{1 / n}\left(\left\|M^{n}\right\|_{1}\right)^{1 / n} \leqslant \rho_{1}(M) .
$$

Since we consider here usual subsets of $A$ as generalized ones, we may calculate $\rho_{1}$ for them (if they are summable). Of course in these calculations the power of a subset must be understood in the above sense (that is, we multiply subsets as generalized subsets).

A normed algebra $A$ is called 1-quasinilpotent if $\rho_{1}(M)=0$ for every summable subset $M$ of $A$. Multiplying elements of a summable generalized subset $M$ of $A$ by different numbers of modulus 1 , it is easy to point out a summable subset $N$ of $A$ such that $\left\|M^{n}\right\|_{1}=\left\|N^{n}\right\|_{1}$ for every $n \in \mathbb{N}$, in particular $\rho_{1}(M)=\rho_{1}(N)$. So, if $A$ is 1 quasinilpotent then $\rho_{1}(M)=0$ for every summable generalized subset $M$ of $A$.

TheOREM 3.7. For a Banach algebra $A$ the following conditions are equivalent.

(i) A is tensor radical.

(ii) $A$ is 1-quasinilpotent.

(iii) $A \widehat{\otimes} B$ is 1-quasinilpotent, for any Banach algebra $B$.

Proof. The implication (iii) $\Rightarrow$ (i) is evident.

(ii) $\Rightarrow$ (i) Let $T=\sum a_{k} \otimes b_{k} \in A \widehat{\otimes} B$. We may suppose that $\left\|b_{k}\right\|=1$ and $\sum\left\|a_{k}\right\|<\infty$. Thus the generalized subset $M$ with the representative $\left\{a_{k}: k=1, \ldots\right\}$ is summable. By our assumption, $\rho_{1}(M)=0$. Now we have

$$
\left\|T^{n}\right\|=\left\|\sum a_{k_{1}} \ldots a_{k_{n}} \otimes b_{k_{1}} \ldots b_{k_{n}}\right\| \leqslant \sum\left\|a_{k_{1}} \ldots a_{k_{n}}\right\|=\left\|M^{n}\right\|_{1}
$$

and accordingly $\rho(T) \leqslant \rho_{1}(M)=0$.

(i) $\Rightarrow$ (ii) Let $G=\mathcal{S}_{1}(W)$ be the free unital semigroup with a countable set $W=$ $\left\{w_{k}\right\}_{k \geqslant 1}$ of generators. That is, $G=\cup_{m \geqslant 0} W_{m}$, where $W_{0}=1$, the direct product

$$
W_{m}=W \times W \times \cdots \times W
$$

is the set of 'words' $w_{k_{1}} w_{k_{2}} \ldots w_{k_{m}}$ of length $m$, and the multiplication is lexical. Let $B=l^{1}(G)$ be the corresponding semigroup algebra. We show that if $A \widehat{\otimes} B$ is radical then $A$ is 1-quasinilpotent.

Indeed, for any summable (generalized) subset $M$ of $A$ with a representative $\left\{a_{k}\right.$ : $k=1,2, \ldots\}$, we define the element $T_{M} \in A \widehat{\otimes} B$ by

$$
T_{M}=\sum a_{k} \otimes w_{k}
$$

Then

$$
T_{M}^{n}=\sum a_{k_{1}} \ldots a_{k_{n}} \otimes w_{k_{1}} \ldots w_{k_{n}} .
$$

Since $A \widehat{\otimes} l^{1}(G)$ is isometrically isomorphic via the map $\pi:(a \otimes f)(g) \rightarrow f(g) a$ to the Banach algebra $l^{1}(G, A)$ of all summable $A$-valued functions on $G$, one has

$$
\left\|T_{M}^{n}\right\|=\sum\left\|a_{k_{1}} \ldots a_{k_{n}}\right\|=\left\|M^{n}\right\|_{1} \text {. }
$$

It follows that

$$
\rho\left(T_{M}\right)=\rho_{1}(M)
$$

and $\rho_{1}(M)=0$. 
(i) $\Rightarrow$ (iii) Since $A$ is tensor radical, the algebra $(A \widehat{\otimes} B) \widehat{\otimes} C=A \widehat{\otimes}(B \widehat{\otimes} C)$ is radical, for any $B$ and $C$. Hence $A \widehat{\otimes} B$ is tensor radical. Applying to $A \widehat{\otimes} B$ the implication (i) $\Rightarrow$ (ii), we see that $A \widehat{\otimes} B$ is 1-quasinilpotent.

COROllary 3.8. A Banach algebra $A$ is tensor radical (=1-quasinilpotent) if and only if $A \widehat{\otimes} l^{1}(G)$ is radical, where $G$ is a free unital semigroup with countable set of generators. Proof. "Only if" is evident, "if" follows from (3.13).

Thus the problem of 1-quasinilpotence of all radical Banach algebras reduces to the problem of tensor perfectness for a single algebra, namely for $l^{1}(G)$.

Now we characterize $\operatorname{Rad}^{t}(A)$ in terms of $\rho_{1}$.

Theorem 3.9. Let $A \in(B A)$. For $a \in A$ the following conditions are equivalent.

(i) $a \in \operatorname{Rad}^{t}(A)$.

(ii) $\rho_{1}(a M)=0$, for any summable subset $M$ of $A$.

(iii) $a \widehat{\otimes} 1 \in \operatorname{Rad}\left(A \widehat{\otimes} l^{1}(G)\right)$, where $G=\mathcal{S}_{1}(W)$ is the free unital semigroup with countable set $W$ of generators.

Proof. (ii) $\Rightarrow(\mathrm{i})$ Let $B \in(B A)$, and let $T=\sum a_{i} \otimes b_{i} \in A \widehat{\otimes} B$ with $\left\|b_{i}\right\|=1$ and $\sum\left\|a_{i}\right\|<$ $\infty$. Then

$$
(a \otimes b) T=\sum a a_{i} \otimes b b_{i}
$$

for arbitrary $b \in B$. Multiplying by numbers of modulus 1 , one can assume that $a_{i} \neq a_{j}$ if $i \neq j$; Let $M$ be a subset of $A$ consisting of all $a_{i}$. Then $a M$ has the representative $\left\{a a_{i}: i \geqslant 1\right\}$ and

$$
\rho((a \otimes b) T) \leqslant\|b\| \rho_{1}(a M)
$$

(the estimate is similar to (3.10) in the proof of Theorem 3.7). Since $\rho_{1}(a M)=0,(a \otimes b) T$ is quasinilpotent. Since this holds for arbitrary $T \in A \widehat{\otimes} B, a \otimes b \in \operatorname{Rad}(A \widehat{\otimes} B)$. Therefore $a \in \operatorname{Rad}^{t}(A)$.

(i) $\Rightarrow$ (iii) is evident.

(iii) $\Rightarrow$ (ii) Let $M$ be a summable subset of $A$ with a representative $\left\{a_{k}: k \geqslant 1\right\}$. Then the element $T_{a M}=\sum\left(a a_{k} \otimes w_{k}\right)$ of $A \widehat{\otimes} l^{1}(G)$ is quasinilpotent because $T_{a M}=(a \otimes 1) T_{M}$ (see the definition of $T_{M}$ in (3.11)). By (3.13), $\rho_{1}(a M)=0$ and this shows that $a$ satisfies (ii).

TheOREM 3.10. $\operatorname{Rad}^{t}$ is a symmetric uniform tensor HTR on $(B A)$.

Proof. It follows from Theorem 3.6 that to see that $\operatorname{Rad}^{t}$ is an HTR we need only prove the inclusion $I \cap \operatorname{Rad}^{t}(A) \subset \operatorname{Rad}^{t}(I)$. This easily follows from Theorem 3.9. Indeed, if $a \in I \cap \operatorname{Rad}^{t}(A)$ then $\rho_{1}(a M)=0$, for every summable subset of $I$, because it is true for subsets of $A$.

The fact that $\operatorname{Rad}^{t}$ is a tensor radical whenever it is a radical was mentioned before.

Since a (closed) subalgebra of a 1-quasinilpotent algebra is clearly 1-quasinilpotent, $\operatorname{Rad}^{t}$ is uniform. It is clear that $\operatorname{Rad}^{t}$ is symmetric.

COROllary 3.11. $\operatorname{Rad}^{t}(A)$ is the largest tensor radical ideal of a Banach algebra A. Moreover, it is closed and contains all (one-sided, non-necessarily closed) 1-quasinilpotent ideals of $A$. 
Proof. The first part is a consequence of Theorem 3.10 and general properties of a TR (Lemma 2.7). The last statement follows immediately from the part (ii) of Theorem 3.9.

Using the extension property of topological radicals (Theorem 2.9), one obtains Corollary 3.12. Let $A \in(B A)$, and let $I$ be its closed ideal. If $A / I$ and $I$ are 1 quasinilpotent then so is $A$.

Now we will show that $\rho_{1}(M)$ can be calculated in the algebra $A / \operatorname{Rad}^{t}(A)$ for every summable family $M$ of elements of $A$.

THEOREM 3.13. For any summable generalized subset $M$ of elements of a Banach algebra $A$,

$$
\rho_{1}(M)=\rho_{1}\left(M / \operatorname{Rad}^{t}(A)\right) .
$$

Proof. Let $J=\operatorname{Rad}^{t}(A)$ and $B=l^{1}(G)$, where $G$ is the free semigroup with countable set of generators. The ideal $i_{A}(J \widehat{\otimes} B)$ of $A \widehat{\otimes} B$ is contained in $\operatorname{Rad}(A \widehat{\otimes} B)$ and the same is true for its closure $U$. Let $E=T_{M} \in A \widehat{\otimes} B$, where $T_{M}$ is defined by (3.11), and $F=E+U$ the image of $E$ in $(A \widehat{\otimes} B) / U$. Then $\rho(E)=\rho(F)$ since $U \subset \operatorname{rad}(A \widehat{\otimes} B)$. This and (3.13) imply $\rho_{1}(M)=\rho(F)$. Let $\tau_{J}:(A / J) \widehat{\otimes} B \rightarrow(A \widehat{\otimes} B) / U$ be the epimorphism introduced in Lemma 3.3. Then clearly $F=\tau_{J}\left(T_{M / J}\right)$, whence

$$
\rho_{1}(M)=\rho(F)=\rho\left(\tau_{J}\left(T_{M / J}\right)\right) \leqslant \rho\left(T_{M / J}\right)=\rho_{1}(M / J) .
$$

We proved that $\rho_{1}(M) \leqslant \rho_{1}(M / J)$. The converse inequality is evident.

Note that the theorem fails when formulated for usual subsets of $A$ and their "usual" (non-generalized) images in $A / \operatorname{Rad}^{t}(A)$.

3.5. Tensor radical and tensor perfect algebras. Our next result shows that the class of tensor perfect algebras is extension stable.

TheOREM 3.14. If a closed ideal $J$ of a Banach algebra $A$ and the quotient $A / J$ are tensor perfect then $A$ is tensor perfect.

Proof. Let $B$ be a radical Banach algebra and $\pi \in \operatorname{irr}(A \widehat{\otimes} B)$. We have to prove that $\pi=0$. Since the algebra $J \widehat{\otimes} B$ is radical and $i_{A}(J \widehat{\otimes} B)$ is its homomorphic image, $i_{A}(J \widehat{\otimes} B)$ consists of quasinilpotent elements. Being an ideal of $A \widehat{\otimes} B$, it is contained in $\operatorname{Rad}(A \widehat{\otimes} B)$. Therefore the closure $i_{A} \widehat{(J \widehat{\otimes} B)} \subset \operatorname{ker} \pi$. This allows one to define a representation $\pi^{\prime}$ of $(A \widehat{\otimes} B) / i_{A} \widehat{(J \widehat{\otimes} B)}$ with the same range as $\pi$. It follows from Lemma 3.3 that the representation $\pi^{\prime \prime}=\pi^{\prime} \circ \tau_{J}$ of $(A / J) \widehat{\otimes} B$ also has the same image as $\pi$. Therefore we have that $\pi^{\prime \prime} \in \operatorname{irr}((A / J) \widehat{\otimes} B)$. Since $(A / J) \widehat{\otimes} B$ is radical, $\pi^{\prime \prime}=0$ and $\pi=0$.

In what follows we list some classes of tensor radical and tensor perfect algebras. Stronger and more general results will be obtained in the next section and further parts of the project.

LEMMA 3.15. Any nilpotent Banach algebra is tensor radical.

Proof. Evidently follows from the fact that the tensor product of a nilpotent algebra and an arbitrary algebra is nilpotent.

Proposition 3.16. Any Banach algebra with dense socle is tensor perfect. 
Proof. Let $p$ be a minimal idempotent in $A$ (in the sense that $\operatorname{dim}(p A p)=1$ ) and $C=p A$. The ideal $J=C(1-p)$ of $C$ is nilpotent and consequently tensor perfect. The quotient $C / J$, being isomorphic to $C p=p A p$, is one-dimensional and hence tensor perfect. By Theorem 3.14, $C$ is tensor perfect. Let $B$ be a radical Banach algebra. The ideal $i_{A}(C \widehat{\otimes} B)$ of $A \widehat{\otimes} B$ consists of quasinilpotent elements (being a homomorphic image of $C \widehat{\otimes} B$ ). Hence $i_{A}(C \widehat{\otimes} B) \subset \operatorname{Rad}(A \widehat{\otimes} B)$. In particular $p a \otimes b \in \operatorname{Rad}(A \widehat{\otimes} B)$ for all $a \in A, b \in B$. Since any element of $\operatorname{soc}(A)$ is a linear combination of elements of the form paq, where $a \in A$, $p$ and $q$ are minimal idempotents, we have that $a \otimes b \in \operatorname{Rad}(A \widehat{\otimes} B)$, for any $a \in \operatorname{soc}(A)$, $b \in B$. By the density assumption, $a \otimes b \in \operatorname{Rad}(A \widehat{\otimes} B)$, for all $a \in A, b \in B$. This means that $A \widehat{\otimes} B$ is radical.

LEMMA 3.17. A Banach algebra $A$ is tensor perfect iff

$$
i_{B}(A \widehat{\otimes} \operatorname{Rad}(B)) \subset \operatorname{Rad}(A \widehat{\otimes} B),
$$

for every Banach algebra $B$.

Proof. Suppose that $A$ is tensor perfect. Then as above, $i_{B}(A \widehat{\otimes} \operatorname{Rad}(B))$, being a homomorphic image of $A \widehat{\otimes} \operatorname{Rad}(B)$, consists of quasinilpotent elements. Hence this ideal is contained in $\operatorname{Rad}(A \widehat{\otimes} B)$. The converse implication is evident.

The following result can be deduced from [1]. Its proof for the case of unital algebras is similar to one of [1, Theorem 4.4.2]; we present the proof because its 'non-unital' part [1, Corollary 4.4.1] seems to be written too briefly.

LEMmA 3.18. If a Banach algebra $A$ is commutative then, for any Banach algebra $B$,

$$
i_{A}(\operatorname{Rad}(A) \widehat{\otimes} B) \cup i_{B}(A \widehat{\otimes} \operatorname{Rad}(B)) \subset \operatorname{Rad}(A \widehat{\otimes} B)
$$

Proof. Suppose first that $A$ and $B$ are unital. For any $\pi \in \operatorname{irr}(A \widehat{\otimes} B)$, let $\pi_{1}(a)=\pi(a \otimes 1)$, $\pi_{2}(b)=\pi(1 \otimes b)$, then $\pi_{1}(A)$ commutes with $\pi_{1}(A)$ and $\pi_{2}(B)$. This means that $\pi_{1}(A)$ is contained in the centre of $\pi(A \widehat{\otimes} B)$. Strict irreducibility of $\pi$ implies that the centre of $\pi(A \widehat{\otimes} B)$ is one-dimensional, i.e. in fact $\pi_{1}(a)=h(a)$ for a character $h$ of $A$. Hence

$$
\pi\left(\sum a_{n} \otimes b_{n}\right)=\sum h\left(a_{n}\right) \pi_{2}\left(b_{n}\right)=\pi_{2}\left(\sum h\left(a_{n}\right) b_{n}\right) .
$$

Therefore $\pi(A \widehat{\otimes} B)=\pi_{2}(B)$ and $\pi_{2} \in$ irr $B$. It follows that $\pi_{2}(\operatorname{Rad}(B))=0$ and, by $(3.15)$,

$$
\pi\left(i_{B}(A \widehat{\otimes} \operatorname{Rad}(B))\right)=0 .
$$

Similarly, since $h(\operatorname{Rad}(A))=0$, we have that

$$
\pi\left(i_{A}(\operatorname{Rad}(A) \widehat{\otimes} B)\right)=0 .
$$

Since $\pi$ is arbitrary, the equality (3.14) is proved.

In the general case, the ideals $J_{1}=i_{A^{1}}\left(\operatorname{Rad}\left(A^{1}\right) \widehat{\otimes} B^{1}\right)$ and $J_{2}=i_{B^{1}}\left(A^{1} \widehat{\otimes} \operatorname{Rad}\left(B^{1}\right)\right)$ are contained in $\operatorname{Rad}\left(A^{1} \widehat{\otimes} B^{1}\right)$, whence they are topologically nil. Since $A$ and $B$ are complemented subspaces in $A^{1}$ and $B^{1}$ respectively, the algebra $A \widehat{\otimes} B$ is topologically isomorphic to its canonical image $J$ in $A^{1} \widehat{\otimes} B^{1}$ (in particular $J$ is an ideal of $A^{1} \widehat{\otimes} B^{1}$ ). Hence $J_{1} \cap J$ and $J_{2} \cap J$ are topologically nil ideals of $J$ and this clearly implies our assertion.

The following statement is an obvious consequence of our results and Lemma 3.18. 
Corollary 3.19. Any commutative Banach algebra is tensor perfect, and any radical commutative Banach algebra is tensor radical.

4. TRs related to the joint quasinilpotence. Let $A$ be a normed algebra. If $N \subset A$, let $\widetilde{N}$ denote the closure of $N$ in $A$ and $\operatorname{abs}(N)$ the closure of absolutely convex hull of $N$. We define here the (usual) sum and product of subsets $M$ and $N$ of $A$ by

$$
M+N=\{a+b: a \in M, b \in N\}
$$

and

$$
M N=\{a b: a \in M, b \in N\},
$$

respectively; similarly for several subsets and for a power $M^{n}, n \in \mathbb{N}$. The set $\mathcal{S}(M)=$ $\cup_{n \geqslant 1} M^{n}$ is called the semigroup generated by $M$. If $A$ is unital, put $\mathcal{S}_{1}(M)=\mathcal{S}(M) \cup\{1\}$.

In what follows we denote by $\mathcal{M}_{f}(A), \mathcal{M}_{c}(A)$ and $\mathcal{M}_{b}(A)$ the classes of all finite, precompact and bounded subsets of a normed algebra $A$, respectively. For $M \in \mathcal{M}_{b}(A)$, one sets

$$
\|M\|=\sup _{a \in M}\|a\|
$$

(the norm of $M$ ) and

$$
\rho(M)=\inf \left\|M^{n}\right\|^{1 / n} .
$$

Since the norm is submultiplicative,

$$
\rho(M)=\inf \left\|M^{n}\right\|^{1 / n}=\lim \left\|M^{n}\right\|^{1 / n} .
$$

The number $\rho(M)$ is called [18] the joint spectral radius of $M$. It is clear that

$$
\begin{aligned}
\rho(M) & =\rho\left(M^{n}\right)^{1 / n} & & \text { for every } n \in \mathbb{N}, \\
\rho(\lambda M) & =|\lambda| \rho(M) & & \text { for every } \lambda \in \mathbb{C}, \\
\rho(M N) & =\rho(N M) & & \text { for every } N \in \mathcal{M}_{b}(A) .
\end{aligned}
$$

The definition of product of subsets of a normed algebra given here differs from the one for generalized subsets defined earlier (in Subsection 3.4). However calculating $\rho(M)$ by rules of generalized subsets gives the same result as in (4.2) because, for a generalized subset $M$ of $A$, the norm $\|M\|=\sup \left\{\|a\|:(a, t) \in M^{\sharp}\right\}$ does not depend on cardinal numbers and coincides with the norm of the usual set $\left\{a:(a, t) \in M^{\sharp}\right\}$. So we prefer to use (4.1) in what follows whenever possible.

We need the following known properties of $\rho$.

Lemma 4.1. Let $A$ be a normed algebra and $N, M \in \mathcal{M}_{b}(A)$.

(i) Then $\rho(L) \leqslant \rho(M)=\rho(\operatorname{abs}(M))$ for any $L \subset \operatorname{abs}(M)$. In particular $\rho(M)=\rho(\widetilde{M})$.

(ii) If every element of $N$ commutes with every element of $M$ then

$$
\begin{aligned}
& \rho(M \cup N)=\max \{\rho(M), \rho(N)\}, \\
& \rho(M+N) \leqslant \rho(M)+\rho(N), \quad \rho(M N) \leqslant \rho(M) \rho(N) .
\end{aligned}
$$

Proof. (i) The equality $\rho(M)=\rho(\operatorname{abs}(M))$ was proved in [19, Proposition 2.6], the other properties are evident.

(ii) was proved in [19, Lemma 2.13]. 
TheOREM 4.2 ([19, Theorem 3.5]). Let $A$ be a normed algebra, and $V \subset \mathbb{C}$ be open. Let $F$ be a family of analytic functions from $V$ into $A$ such that

$$
\lim _{\mu \rightarrow \lambda} \sup \{\|f(\mu)-f(\lambda)\|: f \in F\}=0
$$

for every $\lambda \in V$ and all $M(\lambda)=\{f(\lambda): f \in F\} \in \mathcal{M}_{b}(A)$. Then $\lambda \mapsto \log \rho(M(\lambda))$ and $\lambda \mapsto \rho(M(\lambda))$ are subharmonic on $V$.

For subharmonicity, see [10]. An important general problem of the joint spectral radius theory is to express $\rho$ for precompact sets of operators in $B(X)$ via their spectral characteristics; see some partial results in $[23,19,20]$.

4.1. Joint quasinilpotent algebras and ideals. A normed algebra $A$ is called finitely quasinilpotent (resp. compactly quasinilpotent; boundedly quasinilpotent) if $\rho(M)=0$, for every $M \in \mathcal{M}_{f}(A)$ (resp. $\left.\mathcal{M}_{c}(A) ; \mathcal{M}_{b}(A)\right)$. For brevity, we write that $A$ is $f$-quasinilpotent (resp. $c$-quasinilpotent; $b$-quasinilpotent).

LEMMA 4.3. Let $A$ be a normed algebra.

(i) If $A$ is c-quasinilpotent or b-quasinilpotent then the same is true for its completion $\bar{A}$.

(ii) Let $\phi: A \rightarrow B$ be a continuous homomorphism. If $A$ is f-quasinilpotent then $\phi(A)$ is $f$-quasinilpotent.

Proof. (i) follows from the fact that any bounded (precompact) subset of $\bar{A}$ is contained in the closure of a bounded (respectively precompact) subset of $A$.

To see (ii) it suffices to note that any $M \in \mathcal{M}_{f}(\phi(A))$ is of the form $\phi(N)$, for some $N \in \mathcal{M}_{f}(A)$, whence $\left\|M^{n}\right\| \leqslant\|\phi\|\left\|N^{n}\right\|$ for every $n \in \mathbb{N}$ and $\rho(M)=0$.

Boundedly quasinilpotent algebras are usually called topologically nilpotent [6, 8, 9]. Part (i) of the previous lemma, for this case, is mentioned, for example, in [14]. For $* \in\{f, c, b\}$, an ideal of a normed algebra is called a $*$-quasinilpotent ideal if it is a *-quasinilpotent algebra.

4.2. Ideals $R_{f}, R_{c}$ and $R_{b}$. We set, for $* \in\{f, c, b\}$,

$$
R_{*}(A)=\left\{a \in A: \rho(\{a\} \cup M)=\rho(M), \quad \forall M \in \mathcal{M}_{*}(A)\right\} .
$$

Clearly the condition $\rho(\{a\} \cup M)=\rho(M)$ can be rewritten in the form

$$
\rho(\{a\} \cup M) \leqslant \rho(M)
$$

because the reverse inequality is evident.

Lemma 4.4. If $a \in R_{*}(A)$ then $\lambda a \in R_{*}(A)$, for any $\lambda \in \mathbb{C}$.

Proof. Indeed,

$$
\begin{aligned}
\rho(\{\lambda a\} \cup M) & =\rho\left(\lambda\left(\{a\} \cup \lambda^{-1} M\right)\right)=|\lambda| \rho\left(\{a\} \cup \lambda^{-1} M\right) \\
& =|\lambda| \rho\left(\lambda^{-1} M\right)=\rho(M) .
\end{aligned}
$$

Lemma 4.5. $a \in R_{*}(A)$ iff $\sup \{\rho(\{\lambda a\} \cup M): \lambda \in \mathbb{C}\}<\infty$ for every $M \in \mathcal{M}_{*}(A)$.

Proof. The function $f(\lambda)=\rho(\{\lambda a\} \cup M)$ is subharmonic by Theorem 4.2 and bounded on $\mathbb{C}$; by $[10]$, it is constant. Hence

$$
\rho(\{a\} \cup M)=f(1)=f(0)=\rho(M) .
$$


Our next lemma shows that one may assume $A$ to be unital.

LEMMA 4.6. $R_{*}(A)=R_{*}\left(A^{1}\right)$.

Proof. Any set $M \in \mathcal{M}_{*}\left(A^{1}\right)$ is contained in a set of the form $N+L$, where $N \in \mathcal{M}_{*}(A)$ and $L \in \mathcal{M}_{*}(\mathbb{C})$. Therefore it suffices to show that the function $\lambda \mapsto \rho(\{\lambda a\} \cup(N+L))$ is bounded, for any $a \in R_{*}(A)$. Without loss of generality, we may assume that $0 \in L$. Hence

$$
\{\lambda a\} \cup(N+L) \subset(\{\lambda a\} \cup N)+L
$$

and, by Lemma 4.1,

$$
\begin{aligned}
\rho(\{\lambda a\} \cup(N+L)) & \leqslant \rho((\{\lambda a\} \cup N)+L) \\
& \leqslant \rho(\{\lambda a\} \cup N)+\|L\|=\rho(N)+\|L\| .
\end{aligned}
$$

By induction we have

LEMMA 4.7. If $N$ is a finite subset of $R_{*}(A)$ then $\rho(N \cup M)=\rho(M)$, for any $M \in$ $\mathcal{M}_{*}(A)$.

LEMMA 4.8. $R_{*}(A)$ is a linear subspace of $A$.

Proof. For $a, b \in R_{*}(A)$, set $N=\{a, b\}$. Since $\{(a+b) / 2\} \cup M \subset \operatorname{abs}(N \cup M)$, we obtain from Lemmas 4.1 and 4.7 that

$$
\rho(\{(a+b) / 2\} \cup M) \leqslant \rho(\operatorname{abs}(N \cup M))=\rho(N \cup M)=\rho(M),
$$

for any $M \in \mathcal{M}_{*}(A)$. This shows that $(a+b) / 2 \in R_{*}(A)$ and it remains to use Lemma 4.4 .

Lemma 4.9. $R_{*}(A)$ is an ideal of $A$.

Proof. By Lemma 4.6, one may assume that $A$ is unital. Let $a \in R_{*}(A), b \in A, M \in$ $\mathcal{M}_{*}(A), \lambda \in \mathbb{C}$. Setting $N=\{\lambda a, b, 1\}$, we get

$$
\rho(N \cup M) \leqslant \beta=\rho(\{b, 1\} \cup M) .
$$

It follows that $\rho\left((N \cup M)^{2}\right) \leqslant \beta^{2}$. But $(N \cup M)^{2}$ contains $\{\lambda a b\} \cup M$. Hence

$$
\sup _{\lambda \in \mathbb{C}} \rho(\{\lambda a b\} \cup M) \leqslant \beta^{2}<\infty,
$$

whence $a b \in R_{*}(A)$ by Lemma 4.5. Similarly $b a \in R_{*}(A)$.

TheOREM 4.10. $R_{*}(A)$ is a closed ideal of $A$.

Proof. Note that if $a \in R_{*}(A), M \in \mathcal{M}_{*}(A)$ then, for any $b \in A$,

$$
\begin{aligned}
\rho(\{(a+b)\} \cup M) & \leqslant \rho(\operatorname{abs}(\{2 a, 2 b\} \cup M)) \\
& =\rho(\{2 a, 2 b\} \cup M))=\rho(\{2 b\} \cup M) \\
& \leqslant\|\{2 b\} \cup M\|=\max \{2\|b\|,\|M\|\} .
\end{aligned}
$$

Now if $c \in \widehat{R_{*}(A)}$ then there are $a \in R_{*}(A), b \in A$ with $c=a+b,\|b\|<\|M\| / 2$. Therefore

$$
\rho(\{c\} \cup M) \leqslant \max \{2\|b\|,\|M\|\}=\|M\| .
$$

Changing $c$ by $\lambda c$ and applying Lemma 4.5, we conclude that $c \in R_{*}(A)$ and $R_{*}(A)$ is closed. 
4.3. Properties of the ideals $R_{f}, R_{c}$ and $R_{b}$. Clearly all $R_{*}(A)$ are topologically nil ideals of a normed algebra $A$. Hence, for any normed algebra $A, R_{*}(A) \subset \operatorname{rad}_{n}(A)$ by Theorem 2.17 (ii) or, more transparently,

$$
R_{b}(A) \subset R_{c}(A) \subset R_{f}(A) \subset \operatorname{rad}_{n}(A) .
$$

Moreover

$$
R_{c}(A) \subset \operatorname{Rad}^{\prime}(A) .
$$

This is a consequence of the following 'regularity' property:

Lemma 4.11. Let $A$ be a normed algebra. Then

(i) $R_{b}(A)=A \cap R_{b}(\bar{A})$.

(ii) $R_{c}(A)=A \cap R_{c}(\bar{A})$.

Proof. The statements follow from Lemma 4.3 and the fact that any bounded (resp. precompact) subset of $\bar{A}$ is contained in the closure of a bounded (resp. precompact) subset of $A$.

Lemma 4.12. Let $* \in\{c, b\}$, and let $A$ be a normed algebra. If $N$ is a precompact subset of $R_{*}(A)$ then $\rho(N \cup M)=\rho(M)$, for any $M \in \mathcal{M}_{*}(A)$.

Proof. Let $G(\lambda)=\lambda N \cup M$, for $\lambda \in \mathbb{C}$. By [17, Lemma 8.2.2], there is a sequence $\left(a_{n}\right)$ in $R_{c}(A)$ convergent to 0 with $N \subset$ abs $\left\{a_{n}: n \in \mathbb{N}\right\}$. Let $F_{m}=\left\{a_{n}: n \geqslant m\right\}$, for $m \in \mathbb{N}$. Then $\left\|F_{m}\right\| \rightarrow 0$ as $m \rightarrow \infty$. By Lemmas 4.1 and 4.7 ,

$$
\begin{aligned}
\rho(G(\lambda)) & =\rho(\lambda N \cup M) \leqslant \rho\left(\lambda\left(\operatorname{abs} F_{1}\right) \cup M\right) \leqslant \rho\left(\operatorname{abs}\left(\lambda F_{1} \cup M\right)\right) \\
& =\rho\left(\lambda F_{1} \cup M\right)=\rho\left(\left\{\lambda a_{1}, \ldots, \lambda a_{m-1}\right\} \cup \lambda F_{m} \cup M\right) \\
& =\rho\left(\lambda F_{m} \cup M\right) \leqslant\left\|\lambda F_{m} \cup M\right\|=\max \left\{|\lambda|\left\|F_{m}\right\|,\|M\|\right\}
\end{aligned}
$$

for every $m \in \mathbb{N}$. Take $m$ such that $\left\|F_{m}\right\|<|\lambda|^{-1}$. Then

$$
\rho(G(\lambda)) \leqslant \max \{1,\|M\|\}
$$

for every $\lambda \in \mathbb{C}$. Being a subharmonic function on $\mathbb{C}$ by Theorem $4.2, \lambda \mapsto \rho(G(\lambda))$ is constant. Hence

$$
\rho(M)=\rho(G(0))=\rho(G(1))=\rho(N \cup M) .
$$

Proposition 4.13. Let $A$ be a normed algebra. Then

(i) $R_{f}(A)$ is f-quasinilpotent.

(ii) $R_{c}(A)$ is c-quasinilpotent.

Proof. The statements follow trivially from Lemmas 4.7 and 4.12 .

The ideal $R_{b}(A)$ is not necessarily $b$-quasinilpotent. To show this, let us look at the case that $A$ is commutative.

Proposition 4.14. Let $A$ be a commutative normed $Q$-algebra. Then $\operatorname{rad}(A)=R_{b}(A)$. Proof. By Theorem 2.18 and (4.4), it suffices to show that $\operatorname{rad}(A) \subset R_{b}(A)$. If $a \in \operatorname{rad}(A)$ and $M \in \mathcal{M}_{b}(A)$ then

$$
\rho(\{\lambda a\} \cup M)=\max \{\rho(\lambda a), \rho(M)\}=\rho(M)
$$

by Lemma 4.1 , for every $\lambda \in \mathbb{C}$, whence $a \in R_{b}(A)$ by Lemma 4.5 . 
It is known that there exist commutative radical Banach algebras which are not $b$ quasinilpotent, for example the norm-closed algebra generated by the Volterra integration operator (see [14], where many other examples can be found). On the other hand, each commutative radical Banach algebra $A$ is $c$-quasinilpotent because it follows from the above proposition that $R_{c}(A)=\operatorname{Rad}(A)$.

Recall that $\mathcal{S}_{1}(M)$ denotes the unital semigroup generated by a subset $M$ of a normed algebra.

THEOREM 4.15. Let $A$ be a unital normed algebra and $N, M \subset \mathcal{M}_{b}(A)$. If $N \mathcal{S}_{1}(M)$ is bounded and $\rho\left(N \mathcal{S}_{1}(M)\right)=0$ then $\rho(N \cup M)=\rho(M)$.

Proof. Let $G=\mathcal{S}_{1}(M)$ and $\gamma=\max \{\|M\|, 1\}$. By the assumption, for any $\varepsilon>0$ there is $\beta=\beta(\varepsilon)$ such that

$$
\left\|(N G)^{n}\right\| \leqslant \beta \varepsilon^{n},
$$

for all $n$. Given a number $\lambda \in \mathbb{C}$, set $E_{\lambda}=\lambda N \cup M$. We choose $\varepsilon<|\lambda|^{-1}$. Let $x=$ $x_{1} x_{2} \ldots x_{n} \in\left(E_{\lambda}\right)^{n}$ and let exactly $k$ of the elements $x_{j}$ belong to $\lambda N$. Then

$$
x=\lambda^{k} z y_{1} y_{2} \ldots y_{k},
$$

where all $y_{i} \in N G, z \in G$ and $\|z\| \leqslant \gamma^{n}$. It follows that

$$
\|x\| \leqslant|\lambda|^{k} \beta \varepsilon^{k} \gamma^{n} \leqslant \beta \gamma^{n} .
$$

This shows that $\left\|\left(E_{\lambda}\right)^{n}\right\| \leqslant \beta \gamma^{n}$ for all $n$, whence

$$
\rho\left(E_{\lambda}\right) \leqslant \gamma
$$

for every $\lambda \in \mathbb{C}$. Being a subharmonic function on $\mathbb{C}, \lambda \mapsto \rho\left(E_{\lambda}\right)$ is constant, whence $\rho\left(E_{1}\right)=\rho\left(E_{0}\right)$ which is what we need.

Lemma 4.16. Let $* \in\{c, b\}$, and let $J$ be an (one-sided) *-quasinilpotent ideal of a normed algebra $A$. If $N \in \mathcal{M}_{*}(J)$ then $\rho(N \cup M)=\rho(M)$, for any $M \in \mathcal{M}_{*}(A)$.

Proof. Let $J$ be a right ideal of $A$. One may suppose that $A$ is unital. Suppose first that $\|M\|<1$. Then $N \mathcal{S}_{1}(M) \in \mathcal{M}_{*}(J)$, so that $\rho\left(N \mathcal{S}_{1}(M)\right)=0$. By Theorem 4.15,

$$
\rho(N \cup M)=\rho(M) \text {. }
$$

If $\|M\| \geqslant 1$, take $t>\|M\|$. Then

$$
\rho(N \cup M)=t \rho\left(t^{-1} N \cup t^{-1} M\right)=t \rho\left(t^{-1} M\right)=\rho(M) .
$$

If $J$ is a left ideal of $A$, use (4.3) with the same argument.

Lemma 4.17. Let $* \in\{f, c, b\}$, and let $A$ be a normed algebra. Suppose that $J$ is $R_{*}(A)$ for $* \in\{f, c\}$ or an (one-sided) b-quasinilpotent ideal of $A$ for $*=b$. If $M, N \in \mathcal{M}_{*}(A)$ and $M \subset N+J$ then $\rho(M) \leqslant \rho(N)$.

Proof. For any $a \in M$, denote by $a^{\prime}$ an element of $J$ such that $a-a^{\prime} \in N$. Set $K=\left\{a^{\prime}\right.$ : $a \in M\}$ and, for any $\lambda \in \mathbb{C}, N(\lambda)=\left\{a-\lambda a^{\prime}: a \in M\right\}$. Then the sets $K$ and $N(\lambda)$ are in $\mathcal{M}_{*}(A), K \subset J$ and

$$
N(1) \subset N
$$


The function $f(\lambda)=\rho(N(\lambda))$ is subharmonic on $\mathbb{C}$ by Theorem 4.2. Since $N(\lambda) \subset$ $2 \operatorname{abs}(\lambda K \cup M)$, we have, by Lemma 4.1 and one of Lemmas $4.7,4.12$ and 4.16 corresponding to $* \in\{f, c, b\}$,

$$
\rho(N(\lambda)) \leqslant 2 \rho(\operatorname{abs}(\lambda K \cup M))=2 \rho(\lambda K \cup M)=2 \rho(M) .
$$

Thus $f(\lambda)$ is constant and

$$
\rho(M)=f(0)=f(1)=\rho(N(1)) \leqslant \rho(N) .
$$

Recall that if $J$ is a closed ideal of $A$ then, for any $M \subset A$, we denote by $M / J$ the image of $M$ under the canonical epimorphism $q_{J}: A \rightarrow A / J$.

THEOREM 4.18. Let $* \in\{f, c, b\}$, and let $A$ be a normed algebra. Suppose that $J$ is $R_{*}(A)$ for $* \in\{f, c\}$ or a closed b-quasinilpotent ideal of $A$ for $*=b$. Then $\rho(M)=\rho(M / J)$ for every $M \in \mathcal{M}_{*}(A)$.

Proof. Let $M \in \mathcal{M}_{*}(A)$. For any $\varepsilon>0$, take $n=n(\varepsilon)$ such that $\left\|M^{n} / J\right\|^{1 / n} \leqslant \rho(M / J)+$ $\varepsilon$. It is a general geometric fact that, for arbitrary $\delta>0$, there are sets $N$ and $Q$ in $\mathcal{M}_{*}(A)$ with $M^{n} \subset N+Q, Q \subset J$ and $\|N\| \leqslant\left\|M^{n} / J\right\|+\delta$ (note that this is evident for $*=f$ and $*=b$, the proof for $*=c$ may be found in [19, Lemma 6.9]). Hence, by Lemma 4.17,

$$
\rho\left(M^{n}\right) \leqslant \rho(N+Q) \leqslant \rho(N) \leqslant\|N\| \leqslant\left\|M^{n} / J\right\|+\delta .
$$

Since $\delta$ is arbitrary,

$$
\rho(M)^{n}=\rho\left(M^{n}\right) \leqslant\left\|M^{n} / J\right\| \leqslant(\rho(M / J)+\varepsilon)^{n},
$$

whence $\rho(M) \leqslant \rho(M / J)+\varepsilon$. It follows that

$$
\rho(M) \leqslant \rho(M / J) \text {. }
$$

The converse inequality is evident.

It should be noted that the theorem does not hold for $J=R_{b}$ and every $M \in \mathcal{M}_{b}(A)$ (see (4.8) in the end of this subsection). However, this theorem implies the equality

$$
\rho(M)=\rho\left(M / R_{b}(A)\right),
$$

for every precompact subset $M$ of $A$. Indeed, since $R_{b}(A) \subset R_{c}(A)$, we have that

$$
\rho(M)=\rho\left(M / R_{c}(A)\right) \leqslant \rho\left(M / R_{b}(A)\right) \leqslant \rho(M) .
$$

The next lemma shows in particular that elements of $R_{*}(A)$ have the properties similar to ones of elements of the Jacobson radical.

Lemma 4.19. Let $* \in\{f, c, b\}$, and let $A$ be a normed algebra. Suppose that $J$ is $R_{*}(A)$ for $* \in\{f, c\}$ or a b-quasinilpotent ideal of $A$ for $*=b$. Then $\rho(N M)=0$ and $\rho(N+M)=$ $\rho(M)$ if one of the following conditions holds.

(i) $\quad N \in \mathcal{M}_{*}(J)$ and $M \in \mathcal{M}_{*}(A)$.

(ii) $N$ is a precompact subset of $R_{b}(A)$ and $M$ is a bounded subset of $A$.

Proof. Let (i) hold. Then $N+M \in \mathcal{M}_{*}(A)$. One may suppose that $J$ is closed by Lemma 4.3 in the case $*=b$. It follows from Theorem 4.18 that

$$
\rho(N+M)=\rho((N+M) / J)=\rho(M / J)=\rho(M) .
$$


Furthermore, since $N M \subset(N \cup M)^{2}$,

$$
\rho(N M) \leqslant \rho\left((N \cup M)^{2}\right)=\rho(N \cup M)^{2}=\rho(M)^{2}
$$

by one of Lemmas $4.7,4.12$ and 4.16 corresponding to $* \in\{f, c, b\}$. Changing $N$ to $\lambda N$ we get that

$$
\rho(N M)=0 .
$$

Now let (ii) hold. Note that (4.6) and (4.7) are valid since $\rho(\lambda N \cup M)=\rho(M)$ by Lemma 4.12. Further, if $G(\lambda)=M \cup(\lambda N+M)$ then $G(\lambda) \subset 2 \operatorname{abs}(\lambda N \cup M)$, whence

$$
\rho(G(\lambda)) \leqslant 2 \rho(\operatorname{abs}(\lambda N \cup M))=2 \rho(\lambda N \cup M)=2 \rho(M)
$$

by Lemma 4.12. Since the function $\lambda \mapsto \rho(G(\lambda))$ is bounded and subharmonic on $\mathbb{C}$, it is constant, whence

$$
\rho(N+M) \leqslant \rho(G(1))=\rho(G(0))=\rho(M) .
$$

Changing $M$ to $-(N+M)$ in that inequality, we obtain that

$$
\rho(M) \leq \rho(N-(N+M)) \leqslant \rho(-(N+M))=\rho(N+M),
$$

whence $\rho(N+M)=\rho(M)$.

TheOrem 4.20. Let $A$ be a normed algebra. An element $a \in A$ belongs to $R_{b}(A)$ (resp. $\left.R_{c}(A)\right)$ iff $\rho(a M)=0$, for any bounded (resp. precompact) set $M \subset A$.

Proof. Let $* \in\{c, b\}$. If $a \in R_{*}(A)$ then $\rho(a M)=0$, for each $M \in \mathcal{M}_{*}(A)$ by Lemma 4.19.

Proving the converse implication, suppose first that $\|M\|<1$. In this case $\mathcal{S}_{1}(M) \in$ $\mathcal{M}_{*}\left(A^{1}\right)$ and, moreover, $\mathcal{S}_{1}(M) a \mathcal{S}_{1}(M) \in \mathcal{M}_{*}(A)$. Hence

$$
\rho\left(a \mathcal{S}_{1}(M)\right)^{2}=\rho\left(\left(a \mathcal{S}_{1}(M)\right)^{2}\right)=\rho\left(a \mathcal{S}_{1}(M) a \mathcal{S}_{1}(M)\right)=0
$$

whence $\rho\left(a \mathcal{S}_{1}(M)\right)=0$. By Theorem 4.15,

$$
\rho(\{a\} \cup M)=\rho(M) .
$$

For $\|M\| \geqslant 1$, we take $t>\|M\|$ and get

$$
\rho(\{a\} \cup M)=t \rho(\{a / t\} \cup(1 / t) M)=t \rho((1 / t) M)=\rho(M) .
$$

Corollary 4.21. Let $* \in\{c, b\}$, and let $A$ be a normed algebra. Then $R_{*}(A)$ contains all (one-sided) *-quasinilpotent ideals of $A$ and $R_{c}(A)$ is the largest c-quasinilpotent ideal of $A$.

Proof. Note that $R_{*}(A)$ contains all (one-sided) *-quasinilpotent ideals of $A$ by Lemma 4.16 , and $R_{c}(A)$ is $c$-quasinilpotent itself by Proposition 4.13 , thus it is the largest $c$ quasinilpotent ideal of $A$.

The following example shows that a Banach algebra need not have the largest $b$ quasinilpotent ideal. Let $A_{n}$ denote the algebra of all strictly upper-triangular matrices on $n$-dimensional Hilbert space $H_{n}$, for every $n \in \mathbb{N}$, and let $A$ be the norm closure of the algebraic direct sum $B$ of all algebras $A_{n}$ acting on the Hilbert space $H=\oplus H_{n}$. Clearly $B$ is a union of nilpotent ideals, whence the largest $b$-quasinilpotent ideal would be equal 
to $A$; in particular $A=R_{b}(A)$ by Corollary 4.21 . On the other hand, if $M$ is the unit ball of $A$ then $\left\|M^{n}\right\|=1$, for all $n$, hence $A$ is not $b$-quasinilpotent. In particular

$$
1=\rho(M) \neq \rho\left(M / R_{b}(A)\right)=0 .
$$

4.4. Radical-like properties of ideals $R_{f}, R_{c}$ and $R_{b}$

Lemma 4.22. Let $A$ be a normed algebra and I its ideal. Then

(i) $I \cap R_{f}(A) \subset R_{f}(I)$.

(ii) $I \cap R_{*}(A)=R_{*}(I)$, for $* \in\{c, b\}$.

Proof. The inclusions $I \cap R_{*}(A) \subset R_{*}(I)$ are evident.

Furthermore, let $a \in R_{*}(I)$ and $M \in \mathcal{M}_{*}(A)$, where $* \in\{b, c\}$. Then $(a M)^{2}=a N$, where $N=M a M \in \mathcal{M}_{*}(I)$. Hence $\rho\left((a M)^{2}\right)=0$ implies $\rho(a M)=0$, whence $a \in R_{*}(A)$ by Theorem 4.20 . We proved that $R_{*}(I) \subset R_{*}(A)$. So $R_{*}(I) \subset R_{*}(A) \cap I$.

LEMmA 4.23. Let $A$ be a normed algebra. Then

(i) $R_{*}\left(R_{*}(A)\right)=R_{*}(A)$, for $* \in\{f, c, b\}$.

(ii) $R_{*}\left(A / R_{*}(A)\right)=0$, for $* \in\{f, c\}$.

Proof. The statement (i) is evident.

For (ii), let $\widehat{a}=a / R_{*}(A) \in R_{*}\left(A / R_{*}(A)\right)$. For every $M \in \mathcal{M}_{*}(A)$, set $\widehat{M}=M / R_{*}(A)$ then, for $* \in\{f, c\}$,

$$
\rho(\{a\} \cup M)=\rho\left((\{a\} \cup M) / R_{*}(A)\right)=\rho(\{\widehat{a}\} \cup \widehat{M})=\rho(\widehat{M})=\rho(M)
$$

by Theorem 4.18. This shows that $a \in R_{*}(A)$ and accordingly $\widehat{a}=0$.

LEMMA 4.24. If $I$ is a closed ideal of a normed algebra $A$ then $q_{I}\left(R_{*}(A)\right) \subset R_{*}(A / I)$ for each $* \in\{f, b, c\}$.

Proof. Clearly, for any $M \in \mathcal{M}_{*}(A / I)$, there is $N \in \mathcal{M}_{*}(A)$ with $q_{I}(N)=M$. If $a \in$ $R_{*}(A)$ then

$$
\rho\left(\left\{\lambda q_{I}(a)\right\} \cup M\right)=\rho\left(\left\{\lambda q_{I}(a)\right\} \cup q_{I}(N)\right) \leqslant \rho(\{\lambda a\} \cup N)=\rho(N)
$$

for every $\lambda \in \mathbb{C}$. By Lemma 4.5, $q_{I}(a) \in R_{*}(A / I)$.

Summing up the results of the previous lemmas we obtain the central statement of this subsection.

THEOREM 4.25. $R_{f}$ satisfies properties $\left(1^{\circ}\right),\left(2^{\circ}\right),\left(3^{\circ}\right)$ and $\left(4^{\circ}\right)$ (from the definition of a TR in Subsection 2.4), $R_{c}$ is an HTR and $R_{b}$ is a hereditary UTR on $(N A)$.

Proof. The property $\left(3^{\circ}\right)$ clearly holds for all $R_{*}$. The other properties follow from Theorem 4.10 and lemmas of this subsection.

We will apply for brevity the term 'radical' to all $R_{*}$ (keeping in mind that only $R_{c}$ is proved to be a TR on $(N A)$ ). For instance, they are HTRs on the class of all commutative normed $Q$-algebras (see Proposition 4.14).

Proposition 4.26. All $R_{*}$ are uniform and symmetric, $R_{f}$ is strong and not semiregular, $R_{c}$ and $R_{b}$ are regular and not strong. 
Proof. Since a subalgebra of a $R_{*}$-radical algebra is $R_{*}$-radical, uniformity of all $R_{*}$ is obvious. Also, it is clear that all $R_{*}$ are symmetric. The radicals $R_{c}$ and $R_{b}$ are regular (Lemma 4.11) and in particular semi-regular. However $R_{f}$ is not semi-regular. Indeed, there exists a normed algebra $C=\phi(A)$ for some continuous homomorphism $\phi: A \rightarrow B$ from a topologically nilpotent Banach algebra $A$ to a semisimple Banach algebra $B$, and $C$ is dense in $B$ [7, Example 9.3]. Note that $R_{f}(C)=C$ and $R_{f}(\bar{C})=0$. On the other hand, the same example shows that $R_{c}$ and $R_{b}$ are not strong radicals (in virtue of $A=R_{c}(A)=R_{b}(A)$ and $R_{b}(C) \subset R_{c}(C) \subset R_{c}(\bar{C}) \subset \operatorname{Rad}(B)=0$ ), while $R_{f}$ is strong by Lemma $4.3(\mathrm{ii})$.

As a consequence of regularity, $R_{c}$ and $R_{b}$ are topologically characteristic on $(N A)$ by Theorem 2.31. Is $R_{f}$ topologically characteristic on $(N A)$ ?

TheOREM 4.27. $R_{c}<R_{f}$ on $(N A)$ and $R_{b}<R_{c}$ on $(B A)$. Moreover, $R_{b}$ is not a TR on $(B A)$.

Proof. Proposition 4.13 shows that to see the distinction between $R_{f}$ and $R_{c}$ it suffices to construct an $f$-quasinilpotent algebra which is not $c$-quasinilpotent. Let $\phi: A \rightarrow B$ be the continuous homomorphism of a radical Banach algebra $A$ onto a dense subalgebra $C$ of a semisimple Banach algebra $B$ constructed by P. G. Dixon [7, Example 9.3]. Recall that $A$ is topologically nilpotent, i.e. $b$-quasinilpotent, while $C$ is $f$-quasinilpotent (by Lemma 4.3) and not $c$-quasinilpotent (since its completion is not radical).

Let us prove that $R_{b}<R_{c}$ on $(B A)$. Let $G$ be the free non-unital semigroup with countable set $\left\{x_{0}, x_{1}, \ldots\right\}$ of generators. Let $J$ be the ideal of $G$ consisting of all words which are not subwords of the infinite word

$$
W=x_{0} x_{1} x_{0} x_{2} \ldots x_{0} x_{n-1} x_{0} x_{n} \ldots
$$

( $P$ is a subword of $W$ if $W=R P H$, where $R \in G$ and $H$ is an infinite word). Then $l^{1}(J)$ is isometrically imbedded as an ideal into $l^{1}(G)$. Set $A=l^{1}(G) / l^{1}(J)$ and denote by $X_{i}$ the images of the generators $x_{i}$ in $A$. Note that $X_{0} X_{0}=0$ and $X_{i} P X_{i}=0$ for every monomial $P$ and $i>0$. Hence $X_{i} A X_{i}=0$ and

$$
\left(X_{i} M\right)^{2}=0
$$

for every bounded subset $M \subset A$ and $i>0$. It follows from Theorem 4.20 that

$$
X_{i} \in R_{b}(A) \subset R_{c}(A)
$$

for all $i>0$. Let $J$ be the closed ideal of $A$ generated by $N=\left\{X_{i}: i>0\right\}$. By Lemma 4.22 ,

$$
J=R_{b}(J)=R_{c}(J)
$$

Further, $A / J$ is a one-dimensional algebra generated by a nilpotent. So

$$
A / J=R_{b}(A / J)=R_{c}(A / J) .
$$

Since $R_{c}$ is a TR, $A=R_{c}(A)$ by Theorem $2.9(\mathrm{i})$. But $R_{b}(A) \neq A$ since $X_{0} \notin R_{b}(A)$ in virtue of

$$
\left\|\left(X_{0} N\right)^{n}\right\|^{1 / n}=\left\|X_{0} X_{1} \cdots X_{0} X_{n}\right\|^{1 / n}=1
$$


for every $n \in \mathbb{N}$. This simultaneously proves that $R_{b}$ is not a TR on $(B A)$ (otherwise $A=R_{b}(A)$ as in the case of $R_{c}$, a contradiction).

The following questions are important. Do $R_{f}$ and $R_{c}$ coincide on $(B A)$ ? Does $R_{f}$ coincide on $(B A)$ with Rad?

4.5. $\operatorname{Rad}^{t}$ as a TR related to joint quasinilpotence. Since the tensor radical $\operatorname{Rad}^{t}$ is also related to a kind of joint quasinilpotence it is natural to compare $R_{*}$ with $\operatorname{Rad}^{t}$.

Let us first prove that $\operatorname{Rad}^{t}$ can be defined in the same way as $R_{*}$.

TheOREM 4.28. Let $A$ be a Banach algebra. Then $a \in \operatorname{Rad}^{t}(A)$ iff $\rho_{1}(\{a\} \cup M)=\rho_{1}(M)$ for every summable subset $M$ of $A$.

Proof. If $a \in \operatorname{Rad}^{t}(A)$ then, by Theorem 3.13,

$$
\rho_{1}(\{a\} \cup M)=\rho_{1}\left((\{a\} \cup M) / \operatorname{Rad}^{t}(A)\right)=\rho_{1}\left(M / \operatorname{Rad}^{t}(A)\right)=\rho_{1}(M) .
$$

Conversely, if $\rho_{1}(\{a\} \cup M)=\rho_{1}(M)$ then

$$
\rho_{1}\left((\{a\} \cup M)^{2}\right)=\rho_{1}(\{a\} \cup M)^{2}=\rho_{1}(M)^{2}
$$

by (3.8). Since $a M \subset(\{a\} \cup M)^{2}$ as generalized subsets, we have

$$
\rho_{1}(a M) \leqslant \rho_{1}(M)^{2} .
$$

Replacing $a$ by $\lambda a$ (which clearly satisfies the same condition) for $\lambda \in \mathbb{C}$, we get:

$$
|\lambda| \rho_{1}(a M) \leqslant \rho_{1}(M)^{2},
$$

whence $\rho_{1}(a M)=0$ and it remains to apply Theorem 3.9.

Now we will prove that the radical $R_{c}$ is weakly tensor.

ThEOREM 4.29. If $A$ is a compactly quasinilpotent Banach algebra then $A \widehat{\otimes} B$ is compactly quasinilpotent, for any Banach algebra B.

Proof. If $M$ is a precompact set in $A \widehat{\otimes} B$ then by [17, Corollary 7.2.2] there exists a sequence $a_{n} \otimes b_{n}$ with $a_{n} \rightarrow 0, b_{n} \rightarrow 0$ such that $M \subset \operatorname{abs}\left\{a_{n} \otimes b_{n}: n \geqslant 1\right\}$. Hence

$$
\rho(M) \leqslant \rho\left(\operatorname{abs}\left\{a_{n} \otimes b_{n}: n \geqslant 1\right\}\right)=\rho\left(\left\{a_{n} \otimes b_{n}: n \geqslant 1\right\}\right) \leqslant \rho(P \otimes Q),
$$

where $P=\left\{a_{n}: n \geqslant 1\right\}$ and $Q=\left\{b_{n}: n \geqslant 1\right\}$. But

$$
\left\|(P \otimes Q)^{n}\right\|=\left\|P^{n} \otimes Q^{n}\right\| \leqslant\left\|P^{n}\right\|\left\|Q^{n}\right\|
$$

whence $\rho(P \otimes Q) \leqslant \rho(P) \rho(Q)$. In our assumptions $\rho(P)=0$ because $P$ is precompact. Hence $\rho(M)=0$.

It is clear that any summable set $M$ of a normed algebra is precompact and

$$
\rho(M) \leqslant \rho_{1}(M) \leqslant\|M\|_{1} .
$$

Moreover, if $M$ is finite then

$$
\rho_{1}(M) \leqslant k \rho(M),
$$

where $k=\operatorname{card}(M)$. It follows that if $M$ is summable with $\rho_{1}(M)=0$ then $\rho(M)=0$, and if $M$ is finite with $\rho(M)=0$ then $\rho_{1}(M)=0$. 
Corollary 4.30. $R_{c} \leqslant \operatorname{Rad}^{t} \leqslant R_{f}$ on $(B A)$.

Proof. If $a \in \operatorname{Rad}^{t}(A)$ then $\rho_{1}(\{\lambda a\} \cup M)=\rho_{1}(M)$ for every finite subset $M$ of $A$ and $\lambda \in \mathbb{C}$, by Theorem 4.28 , whence

$$
\sup \{\rho(\{\lambda a\} \cup M): \lambda \in \mathbb{C}\} \leqslant \sup \left\{\rho_{1}(\{\lambda a\} \cup M): \lambda \in \mathbb{C}\right\}=\rho_{1}(M) .
$$

By Lemma 4.5, $a \in R_{f}(A)$.

It follows from Theorem 3.2 that if $A=R_{c}(A)$ then $A=\operatorname{Rad}^{t}(A)$. Then

$$
R_{c}(A)=\operatorname{Rad}^{t}\left(R_{c}(A)\right) \subset \operatorname{Rad}^{t}(A)
$$

because $R_{c}(A)$ is a closed ideal of $A$ and $\operatorname{Rad}^{t}$ is a TR on $(B A)$.

So, it can be said that the problem of the tensor radicality of a radical Banach algebra is "intermediate" between the problems of finite quasinilpotence and compact quasinilpotence. It follows from Corollary 4.30 and Lemma 4.11 that

$$
\rho_{1}(M)=0
$$

for every summable subset $M$ of $R_{c}(A)$, for a normed algebra $A$.

4.6. $p$-quasinilpotent algebras. We will conclude this section by considering a natural scale of joint spectral radii, including $\rho$ and $\rho_{1}$ as polar extrema. For a close (but not identical) notion see [15].

Let $A$ be a normed algebra and $p \geqslant 1$. For a generalized subset $M$ of $A$ with a representative $\left(a_{\alpha}\right)_{\alpha \in \Lambda}$, let

$$
\|M\|_{p}=\left(\sum_{\alpha \in \Lambda}\left\|a_{\alpha}\right\|^{p}\right)^{1 / p}
$$

As in the case $p=1,\|M\|_{p}$ does not depend on a representative. Moreover,

$$
\|M N\|_{p} \leqslant\|M\|_{p}\|N\|_{p}
$$

for generalized subsets of $A$ (recall that the product $M N$ and a power $M^{n}$ are defined here by rules of generalized subsets; see Subsection 3.4). A generalized subset $M$ of $A$ is called p-summable if $\|M\|_{p}<\infty$. For a $p$-summable generalized subset $M$ of $A$, it follows from (4.9) that there exists

$$
\rho_{p}(M)=\lim \left(\left\|M^{n}\right\|_{p}\right)^{1 / n}=\inf \left(\left\|M^{n}\right\|_{p}\right)^{1 / n} .
$$

Changing, as usual, sums to suprema for $p=\infty$, one can write that

$$
\rho(M)=\rho_{\infty}(M) .
$$

Clearly any $p_{1}$-summable generalized subset $M$ is $p_{2}$-summable if $p_{1}<p_{2} ;$ moreover

$$
\|M\|_{p_{1}} \geqslant\|M\|_{p_{2}} \text {. }
$$

It follows that

$$
\rho_{p_{1}}(M) \geqslant \rho_{p_{2}}(M) .
$$

A normed algebra $A$ is called $p$-quasinilpotent if $\rho_{p}(M)=0$, for every $p$-summable subset $M$ of $A$. It is clear that if $A$ is $p$-quasinilpotent then $\rho_{p}(M)=0$ for every $p$ summable generalized subset of $A$. 
TheOREM 4.31. Let $A$ be a Banach algebra and $p>1$. Then

(i) If $A$ is p-quasinilpotent then $A$ is 1-quasinilpotent.

(ii) If $A$ is 1-quasinilpotent then $\rho_{p}(M)=0$, for any 1-summable subset $M$ of $A$.

Proof. (i) Note that the case $p=\infty$ follows from Corollary 4.30. Let $p<\infty$. It suffices to prove that $A$ is $\operatorname{Rad}^{t}$-radical. Let $B$ be an arbitrary Banach algebra and $T=\sum a_{k} \otimes b_{k} \in$ $A \widehat{\otimes} B$ with $\sum\left\|a_{k}\right\|\left\|b_{k}\right\|<\infty$. Multiplying by suitable scalars, one may suppose that $\sum\left\|a_{k}\right\|^{p}<\infty$ and $\sum\left\|b_{k}\right\|^{q}<\infty$, where $q=p /(p-1)$. It follows that

$$
\begin{aligned}
\left\|T^{n}\right\| & =\left\|\sum a_{k_{1}} \ldots a_{k_{n}} \otimes b_{k_{1}} \ldots b_{k_{n}}\right\| \\
& \leqslant\left(\sum\left\|a_{k_{1}} \ldots a_{k_{n}}\right\|^{p}\right)^{1 / p}\left(\sum\left\|b_{k_{1}} \ldots b_{k_{n}}\right\|^{q}\right)^{1 / q} \leqslant\left\|M^{n}\right\|_{p} \beta^{n},
\end{aligned}
$$

where $M$ is a generalized subset with the representative $\left(a_{k}\right)_{k \geqslant 1}$ and

$$
\beta=\left(\sum\left\|b_{k}\right\|^{q}\right)^{1 / q}
$$

Thus

$$
\left\|T^{n}\right\|^{1 / n} \leqslant \beta\left(\left\|M^{n}\right\|_{p}\right)^{1 / n}
$$

whence $\rho(T) \leqslant \rho_{p}(M)=0$.

(ii) follows immediately from (4.10).

\section{References}

[1] B. Aupetit, Propriétés spectrales des algèbres de Banach, Lecture Notes in Mathematics 735, Springer-Verlag, Berlin, 1979.

[2] F. F. Bonsall and J. Duncan, Complete Normed Algebras, Springer-Verlag, Berlin, 1973.

[3] N. Bourbaki, Éléments de mathématique, Théories spectrales, Hermann, 1967.

[4] H. G. Dales, Banach Algebras and Automatic Continuity, Clarendon Press, Oxford, 2000.

[5] N. J. Divinsky, Rings and Radicals, Allen and Unwin, London, 1965.

[6] P. G. Dixon, Topologically nilpotent Banach algebras and factorization, Proc. Royal Soc. Edinburgh A 119 (1991), 329-341.

[7] P. G. Dixon, Topologically irreducible representations and radicals in Banach algebras, Proc. London Math Soc. (3) 74 (1997) 174-200.

[8] P. G. Dixon and V. Müller, A note on topologically nilpotent Banach algebras, Studia Math. 102 (1992), 269-275.

[9] P. G. Dixon and G. A. Willis, Approximate identities in extensions of topologically nilpotent Banach algebras, Proc. Royal Soc. Edinburgh A 122 (1992), 45-52.

[10] W. K. Hayman and P. B. Kennedy, Subharmonic Functions, Volume 1, LMS Monographs 9, Academic Press, London, 1976.

[11] I. Kaplansky, Locally compact rings, Amer. Math. J. 70 (1948), 447-459.

[12] E. Kissin and V. S. Shulman, Representations on Krein Spaces and Derivations of $C^{*}$ algebras, Pitman Monographs and Surveys in Pure and Applied Math. 89, Addison Wesley Longman, London, 1997.

[13] T. W. Palmer, Banach Algebras and the General Theory of *-Algebras, Vol. 1, Cambridge Univ. Press, 1994. 
[14] J. R. Peters and R. W. Wogen, Commutative radical operator algebras, J. Operator Theory 42 (1999), 405-424.

[15] V. Yu. Protasov, The generalized joint spectral radius. A geometric approach, Izv. Math. 61 (1997), 995-1030.

[16] C. J. Read, Quasinilpotent operators and the invariant subspace problem, J. London Math. Soc. (2) 56 (1997), 595-606.

[17] A. P. Robertson and W. Robertson, Topological Vector Spaces, Cambridge Univ. Press, 1964.

[18] G.-C. Rota and W. G. Strang, A note on the joint spectral radius, Indag. Math. 22 (1960), 379-381.

[19] V. S. Shulman and Yu. V. Turovskii, Joint spectral radius, operator semigroups and a problem of W. Wojtyński, J. Funct. Anal. 177 (2000) 383-441.

[20] V. S. Shulman and Yu. V. Turovskii, Formulae for joint spectral radii of sets of operators, Studia Math. 149 (2002), 23-37.

[21] A. M. Sinclair, Continuous derivations on Banach algebras, Proc. Amer. Math. Soc. 20 (1969) 166-170.

[22] F. A. Szász, Radicals of Rings, Akadémiai Kiadó, Budapest, 1981.

[23] Yu. V. Turovskii, Volterra semigroups have invariant subspaces, J. Funct. Anal. 162 (1999), 313-323. 